\title{
CENTER MANIFOLDS FOR PARTIALLY HYPERBOLIC SETS WITHOUT STRONG UNSTABLE CONNECTIONS
}

\author{
CHRISTIAN BONATTI ${ }^{1}$ AND SYLVAIN CROVISIER ${ }^{2}$ \\ ${ }^{1}$ Institut de Mathématiques de Bourgogne, CNRS - URM 5584, \\ Université de Bourgogne, Dijon 21004, France (bonatti@u-bourgogne.fr) \\ ${ }^{2}$ Laboratoire de Mathématiques d'Orsay, CNRS - UMR 8628, \\ Université Paris-Sud 11, Orsay 91405, France (Sylvain.Crovisier@math.u-psud.fr)
}

(Received 15 January 2014; revised 9 February 2015; accepted 9 February 2015; first published online 11 March 2015)

Abstract We consider compact sets which are invariant and partially hyperbolic under the dynamics of a diffeomorphism of a manifold. We prove that such a set $K$ is contained in a locally invariant center submanifold if and only if each strong stable and strong unstable leaf intersects $K$ at exactly one point.

Keywords: center manifold; partial hyperbolicity; heteroclinic intersection

2010 Mathematics subject classification: Primary 37D10

Secondary 37D30; 37C29

\section{Introduction}

Ever since the first works on dynamical systems, attempts have been made to reduce the dimension of the system, and many techniques have been developed for that purpose: first integrals (for some conservative systems), Poincaré return maps on transverse sections (for flows), quotients by invariant foliations, and so on. One of these techniques is the famous center manifold theorem; see for instance [12, 23]. Consider a fixed point $x$ of a diffeomorphism $f$ on a manifold $M$ such that the differential $D f(x)$ leaves invariant a splitting $T_{x} M=E^{s s} \oplus E^{c} \oplus E^{u u}$, corresponding to the parts of the spectrum of $D f(x)$ whose moduli are, respectively, strictly less than 1 , equal to 1 , and strictly greater than 1 . Then there exists a locally $f$-invariant manifold $W^{c}$ through $x$ tangent at $x$ to $E^{c}$. Furthermore, the local topological dynamics of $f$ is the product of the restriction $\left.f\right|_{W^{c}}$ by a uniform contraction in the $E^{s s}$ direction and by a uniform dilation in the $E^{u u}$-direction.

\subsection{Main result}

We propose here a generalization of the center manifold theorem where the fixed point $x$ is replaced by an invariant compact set. Recall that a $D f$-invariant splitting $T M=$ $E^{c} \oplus E^{u u}$ defined over an invariant compact set $K$ is partially hyperbolic with strong 
unstable direction $E^{u u}$ if the vectors in $E^{u u}$ are uniformly expanded and the possible expansion in $E^{c}$ is strictly weaker than the expansion in $E^{u u}$. More precisely, there are constants $\lambda_{K}>1$ and $C>0$ such that, for each $x \in K$, each unit vector $u \in E^{c}(x)$, $v \in E^{u u}(x)$, and each $n \geqslant 1$, one has

$$
\left\|D f^{n}(x) v\right\|>C \lambda_{K}^{n} \quad \text { and } \quad\left\|D f^{n}(x) v\right\|>C \lambda_{K}^{n}\left\|D f^{n}(x) u\right\| .
$$

With these hypotheses, any point in $K$ has a well defined strong unstable manifold $W^{u u}(x)$ tangent to $E^{u u}(x)$. It is the set of points whose backward iterates get closer to those of $x$ at almost the same rates as the contraction of the vectors in $E^{u u}$ by the backward iterates of $D f$.

Main Theorem. Let $f$ be a $C^{1}$-diffeomorphism and $K$ a partially hyperbolic compact invariant set such that $\left.T M\right|_{K}=E^{c} \oplus E^{u u}$. Then, the next two properties are equivalent.

(1) There exists a compact $C^{1}$-submanifold $S$ with boundary which

- contains $K$ in its interior,

- is tangent to $E^{c}$ at each point of $K$ (i.e., $T_{x} S=E^{c}(x)$ for each $x \in K$ ),

- is locally invariant: $f(S) \cap S$ contains a neighborhood of $K$ in $S$,

(2) The strong unstable manifold of any $x \in K$ intersect $K$ only at $x$ (i.e., $W^{u u}(x) \cap K=$ $\{x\})$.

Remarks 1.1. (1) In all the text compact manifold with boundary means compact manifold, possibly with boundary.

(2) The submanifold $S$ is in general not unique: if $S^{\prime}$ is another submanifold the intersection $S \cap S^{\prime}$ could be reduced to $K$, as for the center manifolds of a fixed point.

(3) The implication $1 \Rightarrow 2$ in the main theorem is immediate: if $K$ is contained in $S$ and if $x, y \in K$ share the same strong unstable manifold, the points $x^{\prime}=f^{-n}(x), y^{\prime}=$ $f^{-n}(y)$ for $n \geqslant 0$ large belong to a same local strong unstable manifold. The transversality between $T_{x} S=E^{c}(x)$ and $T_{x} W_{x}^{u u}=E^{u u}(x)$ implies that $x^{\prime}=y^{\prime}$, and hence that $x=y$.

(4) After we wrote a first version of this text, Geneviève Raugel mentioned to us that center manifolds for partially hyperbolic invariant sets have been also built before by Chow, Liu, and Yi [3] for flows generated by a vector fields under different assumptions: they require that the set is tangent at each point to its center bundle and that its geometry is 'bounded' (admissibility condition). Our result shows that their second assumption is not necessary, and that the first one can be replaced by a dynamical property on the strong unstable lamination, which is easier to check in practice. Our assumptions are optimal since we get an equivalence.

The compact set $K$ is not necessarily the maximal invariant set in a neighborhood. The corollary below extends the conclusion of the theorem to the maximal invariant set of a neighborhood. 
Corollary 1.2. Under the conclusion of the main theorem, there is a neighborhood $U$ of $K$ such that the maximal invariant set $\Lambda$ in $U$ is contained in the interior of $S$.

We consider now an invariant compact set $K$ having a $D f$-invariant partially hyperbolic splitting in three bundles $\left.T M\right|_{K}=E^{s s} \oplus E^{c} \oplus E^{u u}$, with strong stable, center, and strong unstable directions $E^{s s}, E^{c}$, and $E^{u u}$, respectively. ${ }^{1}$ The strong stable manifolds $W^{s s}(x)$ of the points of $K$ are the strong unstable manifolds, tangent to $E^{s s}(x)$, for $f^{-1}$.

Corollary 1.3. Let $K$ be a compact invariant set of a diffeomorphism $f$, admitting a partially hyperbolic splitting $\left.T M\right|_{K}=E^{s s} \oplus E^{c} \oplus E^{u u}$. Then there is a compact $C^{1}$-submanifold $S$ with boundary which contains $K$ in its interior, is tangent to $E^{c}$ at each point of $K$, and is locally invariant, if and only if the strong stable and strong unstable manifolds of any point $x \in K$ intersect $K$ only at $x$ (i.e., $W^{u u}(x) \cap K=\{x\}=$ $\left.W^{s s}(x) \cap K\right)$.

We will sometimes reformulate the assumptions on $K$ with the following terminology. We will say that a partially hyperbolic set $K$ admits a strong stable or a strong unstable connection if there is $x \in K$ such that $W^{s s}(x)$ or $W^{u u}(x)$, respectively, meets $K$ in a point different from $x$. A compact invariant set $K$, endowed with a partially hyperbolic structure of type $E^{s s} \oplus E^{c}, E^{c} \oplus E^{u u}$, or $E^{s s} \oplus E^{c} \oplus E^{u u}$, has no strong connection if it has no strong stable connection, no strong unstable connection, or no strong stable or strong unstable connection, respectively.

In the previous statements, the locally invariant submanifold $S$ is tangent to the center direction so that it is normally hyperbolic. In particular, it persists by small perturbations.

Corollary 1.4. Under the conclusion of the main theorem, there exists a submanifold with boundary $S^{\prime} \subset S \cap f(S)$ which contains a neighborhood of $K$ in $S$, and there exist a $C^{1}$-neighborhood $\mathcal{U}$ of $f$ and a neighborhood $U$ of $K$ such that, for any $g \in \mathcal{U}$,

- the maximal invariant set $\Lambda_{g}$ of $g$ in $U$ is contained in a submanifold $S_{g}, C^{1}$-close to $S$,

- $S_{g} \cap g\left(S_{g}\right)$ contains a submanifold $S_{g}^{\prime}, C^{1}$-close to $S^{\prime}$,

- $S_{g}$ and $S_{g}^{\prime}$ depend continuously on $g$ for the $C^{1}$-topology.

In order to describe the local dynamics of $f$ and of its perturbations in the neighborhood of $K$ we are therefore reduced to understanding the dynamics restricted to $S$. As $S$ is a $C^{1}$-submanifold, the induced local diffeomorphism (defined in a neighborhood of $K$ in $S$ ) cannot be a priori more regular than $C^{1}$. The standard results on normal hyperbolicity (see [12]) ensure anyway some better smoothness on $S$ when $f$ is more regular. ${ }^{2}$

\footnotetext{
${ }^{1}$ That is, the splittings in two bundles $\left.T M\right|_{K}=\left(E^{s s} \oplus E^{c}\right) \oplus E^{u u}$ and $\left.T M\right|_{K}=\left(E^{u u} \oplus E^{c}\right) \oplus E^{s s}$ are partially hyperbolic for $f$ and $f^{-1}$, respectively (with strong unstable direction $E^{u u}$ and $E^{s s}$, respectively).

${ }^{2}$ We recall that a map is $C^{k, \alpha}$ with $k \in \mathbb{N}$ and $\alpha \in(0,1]$ if it is $C^{k}$ and its $k$ th derivative is $\alpha$-Hölder with locally uniform Hölder constant. In particular, a $C^{1,1}$-map has a Lipschitz derivative. For $r \in[0,+\infty) \backslash \mathbb{N}$, a map is $C^{r}$ if it is $C^{k, \alpha}$ with $r=k+\alpha$.
} 
We say that a partially hyperbolic set $K$ is $r$-normally hyperbolic, $r \geqslant 1$, if there are constants $\lambda_{K}>1$ and $C>0$ such that for each $x \in K$, each $n \geqslant 1$, and each non-zero vector $v^{s} \in E^{s s}, v^{c} \in E^{c}, v^{u} \in E^{u u}$ at $x$, one has

$$
C \lambda_{K}^{n} \frac{\left\|D f^{n}(x) v^{s}\right\|}{\left\|v^{s}\right\|}<\left[\frac{\left\|D f^{n}(x) v^{c}\right\|}{\left\|v^{c}\right\|}\right]^{r}<C^{-1} \lambda_{K}^{-n} \frac{\left\|D f^{n}(x) v^{u}\right\|}{\left\|v^{u}\right\|} .
$$

Corollary 1.5. Under the conclusion of the main theorem,

- if $f$ is $C^{r}$ and $K$ is $r$-normally hyperbolic, then $S$ can be chosen $C^{r}$;

- if $f$ is $C^{r}, r>1$, then $S$ can be chosen $C^{1, \alpha}$ for some $\alpha>0$.

The study of $C^{1}$-diffeomorphisms sometimes uses approximation by more regular diffeomorphisms (see for instance [19]). For this reason, when $f$ and $S$ are only $C^{1}$, we are interested in getting a more regular submanifold, by a $C^{1}$-perturbation of $f$.

Proposition 1.6. Under the conclusion of the main theorem, there exist a neighborhood $U$ of $K$ and a submanifold with boundary $S^{\prime} \subset S \cap f(S)$ containing $U \cap S$ with the following property.

There exist a $C^{\infty}$-diffeomorphism $g$ and some $C^{\infty}$ submanifolds with boundary $S_{g}^{\prime}, S_{g}$ which are arbitrarily close to $f, S^{\prime}$, and $S$ for the $C^{1}$-topology such that

- the maximal invariant set $\Lambda_{g}$ of $g$ in $U$ is contained in $S_{g}$,

$-S_{g} \cap f\left(S_{g}\right)$ contains the submanifold $S_{g}^{\prime}$.

Even if $f$ is a smooth diffeomorphism, we do not know if it is possible to chose $g$ $C^{r}$-close to $f, r>1$, in Proposition 1.6.

\subsection{Dynamical consequences}

Partially hyperbolic dynamics with center dimension equal to 1 . For a compact partially hyperbolic set without strong stable and strong unstable connections, we may obtain a better description of the local dynamics if the dimension of the center direction is very small. The following corollary asserts that, when the center direction is one dimensional, we can perturb the diffeomorphism in order to get a dynamics which is locally of Morse-Smale type.

Corollary 1.7. Let $K$ be a compact invariant set endowed with a partially hyperbolic structure whose center bundle is one dimensional, and assume that $K$ has no strong connection. Then, there is a compact neighborhood $U$ of $K$ and, for any $C^{1}$-neighborhood $\mathcal{U}$ of $f$, there is a diffeomorphism $g \in \mathcal{U}$ such that the maximal invariant set $\Lambda_{g}$ in $U$ satisfies the following.

- The set of periodic orbits in $\Lambda_{g}$ is finite; each periodic point is contained in the interior of $U$ and is hyperbolic.

- The set of non-periodic points in $\Lambda_{g}$ decomposes into finitely many orbits of compact segments; each of them is contained in the transverse intersection of the stable manifold $W^{s}\left(O_{1}\right)$ and of the unstable manifold $W^{u}\left(O_{2}\right)$ of two periodic orbits $O_{1}, O_{2} \in \Lambda_{g}$. 
For the initial dynamics $f$, any forward orbit in $K$ accumulates on a periodic circle or on a periodic orbit, or on a Cantor set (a minimal set conjugated to the return map on a family of sections of an 'exceptional minimal set' for a $C^{1}$-vector field on a compact surface).

This result may be related with an extension by Pujals and Sambarino of Mañé's theorem [13] about the hyperbolicity of one-dimensional endomorphisms to higher-dimensional diffeomorphisms; see [19] and generalizations [8, 20]:

Let $K$ be a compact invariant set of a $C^{2}$ Kupka-Smale diffeomorphism $f$, with a partially hyperbolic splitting $T M_{\mid K}=E^{s s} \oplus E^{c}$, where $E^{c}$ is one dimensional. Consider the maximal invariant set $\Lambda$ of $f$ in a small neighborhood of $K$. Then the chain-recurrent set of $f$ in $\Lambda$ consists of finitely many normally hyperbolic attracting periodic circles, and finitely many hyperbolic sets.

Palis' hyperbolicity conjecture. A conjecture by Palis claims that any diffeomorphism may be $C^{1}$-approximated by Axiom A diffeomorphisms, or by diffeomorphisms which present a homoclinic tangency (a non-transverse intersection between the stable and unstable manifolds of a hyperbolic periodic orbit) or a heterodimensional cycle (two hyperbolic periodic orbits with different stable dimensions linked by two heteroclinic orbits).

This conjecture has been solved on surfaces [19], and our main theorem allows us to generalize in some cases to higher dimensions ${ }^{3}$ (see also $[7, \S 2.7]$ ).

Corollary 1.8. Let $U$ be a filtrating set of a diffeomorphism $f$ such that the maximal invariant set $\Lambda$ of $f$ in $U$ admits a partially hyperbolic splitting whose center bundle has its dimension equal to 2 . We assume furthermore that $\Lambda$ has no strong connection. Then, in any $C^{1}$-neighborhood $\mathcal{U}$ of $f$ there is a diffeomorphism $g \in \mathcal{U}$ verifying one of the two following properties:

- either there is a hyperbolic periodic saddle $x \in U$ of $g$ whose invariant manifolds present a homoclinic tangency along an orbit of a point $y \in U$;

- or $g$ verifies the 'Axiom $A+$ no cycle condition' in $U$ : the chain-recurrent set $\mathcal{R}(g) \cap U$ in $U$ consists in finitely many hyperbolic transitive sets.

The previous conjecture has motivated studies of diffeomorphisms 'far from homoclinic tangency' or 'far from heterodimensional cycles'. For instance, Wen has shown that the minimally non-hyperbolic sets of diffeomorphisms $C^{1}$-far from tangencies and from heterodimensional cycles are partially hyperbolic with a one-dimensional or

\footnotetext{
${ }^{3}$ A point $x$ is called chain recurrent if $f$ admits $\varepsilon$-pseudo orbits starting and ending at $x$, for any $\varepsilon>0$. On the set $\mathcal{R}(f)$ of chain-recurrent points, one defines a equivalence relation as follows: two points $x, y \in \mathcal{R}(f)$ are equivalent if there are $\varepsilon$-pseudo orbits starting at $x$ and ending at $y$, and conversely starting at $y$ and ending at $x$, for any $\varepsilon>0$. The chain-recurrence classes are the equivalence classes of this relation, inducing a partition of $\mathcal{R}(f)$ in invariant compact sets.

A trapping region of a diffeomorphism is an open set $U$ such that $f(\bar{U})$ is contained in $U$. A filtrating set is the intersection of a trapping region $U$ of $f$ with a trapping region $V$ of $f^{-1}$. One fundamental property of the trapping regions is that a chain-recurrence class of $f$ meeting a trapping region is contained in it.
} 
two-dimensional center bundle (see [22], and its global generalization [5]), reducing the conjecture to the partially hyperbolic setting.

The following proposition shows that the hypotheses 'without strong connection' and 'far from heterodimensional cycle' are related. We recall that two hyperbolic periodic orbits are homoclinically related if they belong to a same transitive hyperbolic set; the homoclinic class of a hyperbolic periodic point $p$ is the closure of the union of the transitive hyperbolic sets containing $p$.

Proposition 1.9. Consider a diffeomorphism $f$ and a compact invariant set $K$ admitting a partially hyperbolic structure whose center bundle is one dimensional. If there exists a dense sequence of periodic orbits $\left(O_{n}\right)$ in $K$ that are homoclinically related and whose center Lyapunov exponents is positive and converge to zero, then

- either there are diffeomorphisms arbitrarily $C^{1}$-close to $f$ having a heterodimensional cycle;

- or for any periodic point $x \in K$ homoclinically related to the $O_{n}$ one has $W^{u u}(x) \cap$ $K=\{x\}$.

When the second case is not satisfied, one says that $K$ has a strong unstable connection at the periodic point $x$. Using a connecting lemma one can then by a $C^{1}$-perturbation create a strong unstable homoclinic intersection at $x$, i.e., an intersection between the strong unstable manifold of the orbit of $x$ and its stable manifold. By unfolding this intersection, one can create a strong homoclinic intersection associated to other periodic orbits: some of them have a center exponent close to 0 , and this allows one to create a heterodimensional cycle by another $C^{1}$-perturbation, which implies the proposition (see $[18, \S 2.3 .2]$ and $[7, \S 2.5]$ ).

At the time we obtained Proposition 1.9, it became for us the main motivation for this work. More precisely, one can ask the following question.

Question 1.10. Let $f$ be a diffeomorphism, $\mathcal{U}$ be a $C^{1}$-neighborhood of $f$, and $p$ be a hyperbolic periodic point such that for any $g \in \mathcal{U}$ the homoclinic class $H(p, g)$ of the hyperbolic continuation $p_{g}$ of $p$ admits a partially hyperbolic structure whose center bundle is one dimensional and expanded along the orbit of $p_{g}$. Does one of the following cases hold?

- Either there is $g \in \mathcal{U}$ such that $H(p, g)$ has a strong unstable connection at a periodic point $x$ homoclinically related to $p_{g}$.

- Or there exists a non-empty open subset $\mathcal{V} \subset \mathcal{U}$ such that $H(p, g)$, for any $g \in \mathcal{V}$, has no strong unstable connection.

Assuming that the center bundle of $H(p)$ is not uniformly expanded, one can expect to show that there exist periodic orbits homoclinically related to $p$ whose center exponent is arbitrarily close to 0 . In the first case of question 1.10, Proposition 1.9 gives a heterodimensional cycle after a $C^{1}$-perturbation of $f$. In the second case, the main theorem shows that the dynamics reduces to a submanifold transverse to the strong unstable bundle: the center direction becomes an extremal one-dimensional bundle and [20] contradicts the fact that it is not uniformly expanded. 
A positive answer to question 1.10 would thus be an important progress for Palis' conjecture. It has been obtained recently in [7, Theorem 10] for quasi-attractors, and (with other results, including the present paper) a weak version of the conjecture has been proved: any diffeomorphism may be $C^{1}$-approximated by diffeomorphisms that are essentially hyperbolic or that present a homoclinic tangency or a heterodimensional cycle.

Invariant foliations of surface hyperbolic sets. The local stable set of a hyperbolic set $K$ supports a natural invariant lamination whose leaves are the stable manifolds. It is sometimes useful to extend it as a foliation $\mathcal{L}^{s}$ which is locally invariant: there exists an neighborhood $U$ of $K$ such that, for any $x$ close to $K$, the connected components of $f\left(\mathcal{L}_{x}^{s}\right) \cap U$ and of $\mathcal{L}_{f(x)}^{s} \cap U$ containing $f(x)$ coincide. This has been used for instance in the original works on the Newhouse phenomenon $[14,15]$ and in the proof of the structural stability for hyperbolic surface diffeomorphisms [9]. The following well-known result becomes a simple consequence of our main theorem. It asserts that a $C^{2}$ surface diffeomorphism near a hyperbolic set is ' $C^{1}$-conjugated to a product'.

Corollary 1.11. Let $f$ be a $C^{2}$-surface diffeomorphism and $K$ be an invariant compact set which is hyperbolic. Then, there exists a $C^{1}$-foliation locally invariant in a neighborhood of $K$ which is tangent to the stable bundle of $K$. If moreover $f$ is $C^{r}, r>2$, then the foliation can be chosen $C^{1, \alpha}$, for some $\alpha>0$.

We do not assume that $K$ is the maximal invariant set in a neighborhood. For a classical proof, see [16, Appendix 1].

Newhouse phenomenon in dimension larger than or equal to 3. Newhouse has shown $[14,15]$ that, among $C^{2}$-diffeomorphisms of a surface, the existence of a homoclinic tangency for $f$ generates an open set $\mathcal{U}$ of diffeomorphisms close exhibiting

- persistent tangencies: there exists a transitive hyperbolic set whose local stable and local unstable sets have a non-transverse intersection for any diffeomorphism $g \in \mathcal{U}$ ),

- generic wild dynamics: there exist infinitely many sinks or sources for any diffeomorphism in a dense $\mathrm{G}_{\delta}$ subset of $\mathcal{U}$.

These properties have been generalized to higher dimension by Palis and Viana [17] for diffeomorphisms exhibiting a sectionally dissipative homoclinic tangency, whereas Romero [21] has obtained the first property for diffeomorphisms exhibiting an arbitrary homoclinic tangency. Their proof tries to reduce to the dimension 2 by either building a locally invariant $C^{2}$ surface which support part of the dynamics, or by building an 'intrinsic two-dimensional differentiable structure'.

The second author and Nicolas Gourmelon have noticed [6] that it is possible to recover these results using the two following ingredients.

- After perturbation, the homoclinic tangency satisfies a generic condition and the main theorem can be applied: the dynamics in a neighborhood of the homoclinic tangency is contained in a $C^{1, \alpha}$-surface. This allows one to reduce to the dimension 2 as expected; however, the smoothness of the induced dynamics is a priori less than $C^{2}$. 
- The Newhouse phenomenon on surfaces also holds for $\alpha \in(0,1)$ in the space of $C^{1, \alpha}$-diffeomorphisms whose $C^{1, \alpha}$ norm is bounded, endowed with the $C^{1}$-topology.

\subsection{Strategy of the proof, and structure of the paper}

The Main Theorem is obtained in two steps.

- In $\S 3$, we use Whitney's extension theorem in order to build a submanifold $S$ tangent to the center direction which contains $K$.

- In $\S 4$, we implement a graph transform argument in order to modify this submanifold and get the local invariance.

Section 2 is devoted to classical preliminary results. The corollaries are proved in $\S 5$.

The general strategy of [3] also follows these two steps, but there are two important differences (beyond the fact that we deal with diffeomorphisms). In the first step, we relate the assumptions of Whitney's theorem to the lack of strong connections. In the second step, we implement the graph transform argument in a different way, which explains why the admissibility condition does not appear in our work. A key point in our proof is to choose carefully the neighborhood where the graph transform is defined: it has to be small, and much thinner along the strong directions (see Proposition 4.9).

\section{Preliminaries}

In this section, we recall results about distances to a compact set and dominated splittings.

Notation. In the whole paper, we denote by $L \cdot v$ the image of $v$ by the linear map $L$.

\subsection{Smoothing the distance to a compact set}

We will need to consider a smooth function which evaluates the distance to a compact set.

Proposition 2.1. Let $\Sigma$ be a compact Riemannian manifold with boundary.

Then, there exists a constant $C_{\Sigma}>0$ such that for any disjoint compact sets $K, L \subset$ $\Sigma$ there is a function $\varphi: \Sigma \rightarrow[0,1]$ which is as smooth as the manifold $\Sigma$, such that $\varphi^{-1}(0)=K, \varphi^{-1}(1)=L$, and such that the norm of the differential $D \varphi$ is bounded by $\frac{C_{\Sigma}}{d(K, L)}$, where $d(K, L)=\inf \{d(x, y) \mid x \in K$ and $y \in L\}$.

We first prove the result in $\mathbb{R}^{n}$.

Lemma 2.2. For $n \geqslant 1$, there exists a constant $\Delta(n)>0$ such that for any disjoint compact subsets $K, L \subset \mathbb{R}^{n}$ there is a smooth function $\varphi: \mathbb{R}^{n} \rightarrow[0,1]$ such that $\varphi^{-1}(0)=$ $K, \varphi^{-1}(1)=L$, and whose derivative has a norm bounded by $\Delta(n) d(K, L)$.

Proof. Let us choose $\varepsilon>0$ small, and introduce a smooth function $h:[-(1+\varepsilon), 1+$ $\varepsilon]^{n} \rightarrow[0,1]$ which coincides with zero on a neighborhood of the boundary of the cube $[-(1+\varepsilon), 1+\varepsilon]^{n}$ and with 1 on a neighborhood of $[-1,1]^{n}$. 
For $k \geqslant 0$, let $\mathcal{P}_{k}$ be the dyadic partitions of $\mathbb{R}^{n}$ which is the collection of cubes of the form

$$
C=\left[-2^{-(k+1)}, 2^{-(k+1)}\right]+2^{-k} V,
$$

where $V$ is a vector in $\mathbb{Z}^{n}$. One also defines the larger cubes

$$
\begin{gathered}
C_{\varepsilon}=\left[-2^{-(k+1)}(1+\varepsilon), 2^{-(k+1)}(1+\varepsilon)\right]+2^{-k} V, \\
\widehat{C}=\left[-3 \times 2^{-(k+1)}, 3 \times 2^{-(k+1)}\right]+2^{-k} V .
\end{gathered}
$$

For $k>0$, the cube $\widehat{C}$ is a union of cubes of $\mathcal{P}_{k}$.

We associate to the cube $C$ the function $h: C_{\varepsilon} \rightarrow[0,1]$ defined by

$$
h_{C}: x \mapsto a_{k} h\left((x-V) 2^{k+1}\right),
$$

for some $a_{k} \in\left(0,2^{-k}\right]$. Its derivative is bounded by some constant $D$, uniform in $k$. We choose $a_{0}=1$ so that for $k=0$ the map $h_{C}$ is bounded from below by 1 on $C$.

Let $K \subset \mathbb{R}^{n}$ be a compact set, and consider the collection $\mathcal{C}$ of cubes $C$ which satisfy the following:

$-C \in \mathcal{P}_{k}$ for some $k \geqslant 0$,

- the larger cube $\widehat{C}$ is disjoint from $K$,

- if $k \neq 0$, there is no cube $C^{\prime} \in \mathcal{P}_{k-1}$ containing $C$ such that $\widehat{C}^{\prime}$ is disjoint from $K$.

Note that the cubes of $\mathcal{C}$ cover $U=\mathbb{R}^{n} \backslash K$ and have disjoint interior. Moreover, two cubes $C, C^{\prime} \in \mathcal{C}$ that are adjacent belong to partitions $\mathcal{P}_{k}, \mathcal{P}_{k^{\prime}}$ with $\left|k^{\prime}-k\right| \leqslant 1$. In particular , any point $x \in U$ belongs to at most $2^{n}$ cubes $C_{\varepsilon}$ associated to $C \in \mathcal{C}$.

The function $\varphi_{K}$ defined on $\mathbb{R}^{n} \backslash K$ by

$$
\varphi_{K}=\sum_{C \in \mathcal{C}} h_{C}
$$

is thus positive, smooth, and bounded by $2^{d}$. It is bounded from below by 1 outside the $2 d$-neighborhood of $K$ (this neighborhood is covered by cubes $C \in \mathcal{C} \cap \mathcal{P}_{0}$ ). Its derivative is bounded by $2^{n} D$. Note also that, if the sequence $a_{k}$ decreases fast enough to zero as $k \rightarrow+\infty$, then one can extend $\varphi_{K}$ by 0 on $K$ and get a smooth function of $\mathbb{R}^{n}$.

If $K, L \subset \mathbb{R}^{n}$ are two disjoint compact subsets of $\mathbb{R}^{n}$, one may define

$$
\varphi=\frac{\varphi_{K}}{\varphi_{K}+\varphi_{L}}
$$

which is smooth, has values in $[0,1]$, and satisfies moreover $\varphi^{-1}(K)=0$ and $\varphi^{-1}(L)=1$. Its derivative is bounded by

$$
\|D \varphi\| \leqslant 2 \frac{\left\|D \varphi_{K}\right\|+\left\|D \varphi_{L}\right\|}{\varphi_{K}+\varphi_{L}} .
$$

Let us assume that the distance between $K$ and $L$ is equal to $2 n$. The sum $\varphi_{K}+\varphi_{L}$ is thus bounded from below by 1 everywhere, and $\|D \varphi\|$ is smaller than $\frac{2^{n+2} D}{2 n} d(K, L)$.

One can reduce to the case when the distance from $K$ to $L$ is equal to $2 n$ by taking the image by an homothety. The lemma thus holds for $\Delta(n)=\frac{2^{n+2} D}{2 n}$. 
We now prove the manifold case.

Proof of Proposition 2.1. Let us consider a finite collection of charts $\psi_{i}: U_{i} \rightarrow \mathbb{R}^{n}$ of $\Sigma$, $i=1, \ldots, \ell$, whose union coincides with $\Sigma$. Let us choose a partition of the unity, i.e., some functions $\theta_{i}: U_{i} \rightarrow[0,1]$ such that

$-\sum_{i} \theta_{i}(x)=1$ at any point $x \in \Sigma$,

- for each $i$, the support $Q_{i}$ of $\theta_{i}$ is a compact subset of $U_{i}$.

If $K, L$ are two compact subsets of $\Sigma$, one can consider the function $\widetilde{\varphi}_{i}: \mathbb{R}^{n} \rightarrow[0,1]$ associated to the compact sets $K_{i}:=\psi_{i}\left(K \cap Q_{i}\right)$ and $L_{i}:=\psi_{i}\left(L \cap Q_{i}\right)$ by Lemma 2.2. Note that $\varphi_{i}:=\theta_{i} \times\left(\widetilde{\varphi}_{i} \circ \psi_{i}\right)$ satisfies the following:

$-0<\varphi_{i}(x)<\theta_{i}(x)$ for points of the interior of $Q_{i} \backslash(K \cup L)$,

$-\varphi_{i}(x)=0$ for $x \in K$,

$-\varphi_{i}(x)=\theta_{i}$ for $x \in L$,

- the derivative of $\varphi_{i}$ is bounded by $C_{i} / d\left(\psi_{i}\left(K_{i}, L_{i}\right)\right.$ where $C_{i}$ does not depend on $K, L$.

Since any $x \in \Sigma \backslash(K \cup L)$ belongs to the interior of some $Q_{i}$, one deduces that $\varphi:=\sum_{i} \varphi_{i}$ is equal to 0 on $K$, to 1 on $L$, and has values inside $(0,1)$ elsewhere. Its derivative is bounded by

$$
\|D \varphi\| \leqslant \sum_{i} \frac{C_{i}}{d\left(\psi_{i}\left(K_{i}\right), \psi_{i}\left(L_{i}\right)\right)} \leqslant \sum_{i} \frac{C_{i} \cdot L_{i}}{d\left(K \cap Q_{i}, L \cap Q_{i}\right)},
$$

where $L_{i}$ bounds the Lipschitz constant of $\psi_{i}^{-1}$. The function $\varphi$ is as smooth as the charts $\psi_{i}$ and the manifold $\Sigma$. The proposition thus holds with $C_{\Sigma}=\sum_{i} C_{i} L_{i}$.

\subsection{Cone fields and dominated splitting}

We recall here well-known facts about dominated splitting and cone fields. This section is used in order to control the smoothness of the center manifold, and can be skipped at a first reading.

Definition 2.3. A continuous cone field $\mathcal{C}$ of dimension $d$ is a family of closed cones $\mathcal{C}(x) \subset T_{x} M$ such that

$-\mathcal{C}(x)=\overline{\operatorname{Interior}(\mathcal{C}(x))}$ for the topology on $T_{x} M$;

- for each $x \in M$, there exists a $d$-dimensional subspace contained in $\operatorname{Interior}(\mathcal{C}(x)) \cup$ $\{0\}$ and a $(\operatorname{dim}(M)-d)$-dimensional space disjoint from $\mathcal{C}(x) \backslash\{0\}$;

- the set of unit vectors of $\mathcal{C}(x)$ and the set of unit vectors of $T_{x} M \backslash \operatorname{Interior}(\mathcal{C}(x))$ depend continuously on $x$ for the Hausdorff topology.

The collection of cones $T_{x} M \backslash \operatorname{Interior}(\mathcal{C}(x))$ is a continuous cone field, called the complementary cone field. A $d$-dimensional $C^{1}$-submanifold $S \subset M$ is tangent to $\mathcal{C}$ if $T_{x} S \subset \mathcal{C}(x)$ for each $x \in S$.

The cone field $\mathcal{C}$ is transverse to a submersion $\pi: M \rightarrow \Sigma_{0}$ if, for each $x \in \Sigma_{0}$ and $z \in \pi^{-1}(z)$, the tangent space at $z$ of the fiber $T_{z} \pi^{-1}(x)$ and $\mathcal{C}(z) \backslash\{0\}$ are disjoint. 
2.2.1. Contracted cone fields. The notion of a contracted cone field is usually defined for diffeomorphisms. We allow here non-surjective tangent maps, which will be necessary when we will consider graph transforms.

Let us consider a $C^{1}$-map $\Psi: U \rightarrow M$ defined on an open subset $U \subset M$.

Definition 2.4. For $r \geqslant 1$, the cone field $\mathcal{C}$ is $r$-contracted by $\Psi$ if there exist $\lambda>1$ and $n_{0} \geqslant 1$ such that, for any $n \geqslant n_{0}$, and any $x$ in $U \cap \Psi^{-1}(U) \cap \cdots \cap \Psi^{-n+1}(U)$, we have

- $D \Psi^{n}(x) \cdot \mathcal{C}(x) \subset \mathcal{C}\left(\Psi^{n}(x)\right)$,

- $D \Psi^{n}(x) \cdot u$ is non-zero if $u \in \mathcal{C}(x) \backslash\{0\}$,

- for any unit vectors $u, v \in T_{x} M$ such that $u \in \mathcal{C}(x)$ and $D \Psi^{n}(x) \cdot v \notin \mathcal{C}\left(\Psi^{n}(x)\right)$,

$$
\min \left(\left\|D \Psi^{n}(x) \cdot u\right\|,\left\|D \Psi^{n}(x) \cdot u\right\|^{r}\right)>\lambda^{n}\left\|D \Psi^{n}(x) \cdot v\right\| .
$$

When $r=1$, we simply say that the cone field is contracted.

Remarks 2.5. (1) The second item implies that, if a submanifold $S$ is tangent to $\mathcal{C}$ and invariant by $\Psi$, then the restriction $\Psi_{\mid S}$ is a local diffeomorphism.

(2) We want that an $r$-contracted cone field is also $r^{\prime}$-contracted for any $r^{\prime} \in[1, r]$. This is the reason why the minimum $\min \left(\left\|D \Psi^{n}(x) \cdot u\right\|,\left\|D \Psi^{n}(x) \cdot u\right\|^{r}\right)$ appears in the third item. Up to replacing $U$ by any open set $U^{\prime}$ relatively compact in $U$, a contracted cone field is also $r$-contracted for some $r>1$ (with the same constant $n_{0}$ ).

(3) If $\Psi$ is a diffeomorphism and if $\mathcal{C}$ is contracted, the complementary cone field is contracted by $f^{-1}$.

Let us define, for $n \geqslant 1$ and $z \in \Psi(U) \cap \cdots \cap \Psi^{n}(U)$, the cone $\mathcal{C}^{n}(z):=D \Psi^{n}\left(\Psi^{-n}(z)\right)$. $\mathcal{C}\left(\Psi^{-n}(z)\right)$, and for $x \in U \cap \cdots \cap \Psi^{-n+1}(U)$, the cone $\mathcal{C}^{-n}(x):=D \Psi^{-n}\left(\Psi^{n}(x)\right) \cdot \mathcal{C}\left(\Psi^{n}(x)\right)$. The following lemma justifies that the cone field is contracted.

Lemma 2.6. If the cone field $\mathcal{C}$ is contracted, there exist $C_{1}>0, \lambda>1$ such that, for any $n \geqslant 1$ and $z \in \Psi(U) \cap \cdots \cap \Psi^{n}(U)$, the cone $\mathcal{C}^{n}(z)$ is exponentially thin: there exists ad-dimensional space $F \subset \mathcal{C}^{n}(z)$ and, for any unit vector $u \in \mathcal{C}^{n}(z)$, there is $w \in F$ such that $\|w-u\| \leqslant C_{1} \lambda^{-n}$. Similarly, for $x \in U \cap \cdots \cap \Psi^{-n+1}(U)$, the cone $T_{x} M \backslash \mathcal{C}^{-n}(x)$ is exponentially thin.

Proof. The proof will use the following claim.

Claim 2.7. There exist $m_{0} \geqslant 1$ and $\sigma>0$ such that, for any $n \geqslant m_{0}$, the angle between the vectors $u$ and $v$ in the third item of Definition 2.4 is bounded from below by $\sigma$. The same holds for the angle between $D \Psi^{n}(x) \cdot u$ and $D \Psi^{n}(x) \cdot v$, if this last vector is not zero.

Proof. One chooses $k$ in $\left[m_{0} / 3,2 m_{0} / 3\right]$. If $m_{0}$ is large enough, we have $\lambda^{k}>2$. By invariance of $\mathcal{C}$, we get that $D \Psi^{k}(x) \cdot v \notin \mathcal{C}\left(\Psi^{k}(x)\right)$, so that by the cone contraction $\left\|D \Psi^{k}(x) \cdot u\right\| \geqslant 2\left\|D \Psi^{k}(x) \cdot v\right\|$. Since $\left\|D \Psi^{k}\right\|$ is bounded, this implies that the angle between $u$ and $v$ is bounded from below, proving that the angle between $u$ and $v$ is bounded away from zero when $n \geqslant m_{0}$. A similar argument holds for $D \Psi^{n}(x) \cdot u$ and $D \Psi^{n}(x) \cdot v$. 
Let us prove now the statement of the lemma. We set $x=\Psi^{-n}(z)$. By the definition of the cone field, there exists a $d$-dimensional space $F_{0} \subset \mathcal{C}(x)$ such that $F:=D \Psi^{n}(x) \cdot F_{0}$ is also $d$-dimensional. Similarly, there exists a transverse $(\operatorname{dim}(M)-d)$-dimensional space $E \subset T_{z} M$ which is not contained in $\mathcal{C}(z)$. One can thus decompose any unit vector $u \in$ $\mathcal{C}^{n}(z)$ as $w+v$ with $w \in F$ and $v \in E$. By definition, $u$ has a preimage $u_{0} \in \mathcal{C}(x)$ and $w$ also. Hence, there exists a preimage $v_{0} \in T_{x} M$ of $v=u-w$ by $D \Psi^{n}(x)$. The cone contraction gives, since $u$ is a unit vector,

$$
\|v\| \leqslant \lambda^{-n} \frac{\left\|v_{0}\right\|}{\left\|u_{0}\right\|} .
$$

One may assume that $v_{0} \neq 0$. Since $v_{0}$ is the preimage by $D \Psi^{n}(x)$ of a vector $v \notin \mathcal{C}(z)$ and $u_{0}-v_{0}$ belongs to $\mathcal{C}(x)$, the angle between $u_{0}-v_{0}$ and $v_{0}$ is bounded from below by $\sigma$. One deduces that $\frac{\left\|v_{0}\right\|}{\left\|u_{0}\right\|}$ is uniformly bounded; hence there exists $C_{1}>0$ such that $\frac{\left\|v_{0}\right\|}{\left\|u_{0}\right\|}<C_{1}$. This gives the required estimate.

The argument for $T_{x} M \backslash \mathcal{C}^{-n}(z)$ is very similar after noting that $D \Psi^{-n}(z) \cdot E$ is a $(\operatorname{dim}(M)-d)$-linear space contained in $\left(T_{x} M \backslash \mathcal{C}(x)\right) \cup\{0\}$.

Let us denote by $m\left(D \Psi^{n}(x)\right)$ the infimum of the norms $\left\|D \Psi^{n}(x) \cdot u\right\|$ over unit vectors $u \in T_{x} M$. Here is another consequence of cone contraction.

Lemma 2.8. If the cone $\mathcal{C}$ is contracted, there exists $C_{2}>0$ such that, for any $n \geqslant 1$, any $x \in U \cap \Psi^{-1}(U) \cap \cdots \cap \Psi^{-n+1}(U)$, and any unit vector $u \in T_{x} M$, we have

$$
\left\|D \Psi^{n}(x) \cdot u\right\| \geqslant C_{2} m\left(D \Psi^{n}(x)_{\mid T_{x} M \backslash \mathcal{C}^{-n}(x)}\right) .
$$

Proof. Any unit vector $u \in T_{x} M$ decomposes as $u=u_{1}+u_{2}$ such that $u_{1} \in \mathcal{C}(x)$ and $u_{2} \in T_{x} M \backslash \mathcal{C}^{-n}(x)$. By Claim 2.7, the angle between $D \Psi^{k} \cdot u_{1}$ and $D \Psi^{k} \cdot u_{2}$ is uniformly bounded away from zero for any $k \in\{0, \ldots, n\}$ (unless one of these vectors is zero). As a consequence,

$$
\left\|D \Psi^{n} \cdot u\right\| \geqslant \widetilde{C} \max \left(m\left(D \Psi_{\mid \mathcal{C}(x)}^{n}\right)\left\|u_{1}\right\|, m\left(D \Psi_{\mid T_{x} M \backslash \mathcal{C}^{-n}(x)}^{n}\right)\left\|u_{2}\right\|\right) .
$$

This concludes the proof after noting that $\max \left(\left\|u_{1}\right\|,\left\|u_{2}\right\|\right)$ is bounded away from below and that $m\left(D \Psi_{\mid \mathcal{C}(x)}^{n}\right) \geqslant m\left(D \Psi_{\mid T_{x} M \backslash \mathcal{C}^{-n}(x)}^{n}\right)$ by the cone contraction.

2.2.2. Dominated splitting. In order to prove higher smoothness in Corollary 1.5, we extend the usual definition of dominated splitting to the notion of $r$-dominated splitting. It is related to the dominated splitting as the $r$-hyperbolicity in [12] is related to the hyperbolicity.

The existence of a dominated splitting and of a contracted cone field are two close properties.

Definition 2.9. Let us consider an invariant compact set $K$ for a diffeomorphism $f$ and an invariant splitting $T M_{\mid K}=E \oplus F$. We say that $E$ is $r$-dominated by $F$ if there exist $C^{\prime}>0, \lambda>1$, and $n \geqslant 1$ such that for any unit vectors $u \in E(x)$ and $v \in F(x)$ we have

$$
\max \left(\left\|D f^{n}(x) \cdot u\right\|,\left\|D f^{n}(x) \cdot u\right\|^{r}\right)<C^{\prime-1} \lambda^{-n}\left\|D f^{n}(x) v\right\| .
$$


One can extend the bundles $E^{c}, E^{u u}$ as two (non-invariant) continuous bundles $E, F$ over a neighborhood $U$ of $K$. For any $x \in U$ and any $\beta>0$, one defines the cone field associated to the splitting $E \oplus F$ and to the Riemannian metric:

$$
\mathcal{C}_{\beta}(x)=\left\{w \in T_{x} M \mid \exists u \in E(x), \exists v \in F(x), w=u+v,\|u\| \leqslant \beta\|v\|\right\} .
$$

For $\beta^{\prime}<\beta$ and $n \geqslant 1$ such that $C^{\prime} \lambda^{-n} \beta<\beta^{\prime}$, we have, for any $x$ close to $K$,

$$
D f^{n}\left(\mathcal{C}_{\beta}(x)\right) \subset \mathcal{C}_{\beta^{\prime}}(f(x)) .
$$

Lemma 2.10. If $\Psi$ is a diffeomorphism between $U$ and its image, if $K \subset U$ is an invariant compact set, and $d \geqslant 1$ an integer, there exists a dominated splitting $T M_{\mid K}=E \oplus F$ with $d=\operatorname{dim}(F(x))$ for each $x \in K$, if and only if there exists a contracted cone field of dimension $d$ on a neighborhood of $K$.

The bundle $F$ is $r$-dominated by $E$ for $f=\Psi^{-1}$ if and only if there exists a cone field of dimension $d$ on a neighborhood of $K$ which is $r$-contracted by $\Psi$.

Proof. The contracted cone field can be defined from a dominated splitting as in (1). Conversely, if there exists a contracted cone field, $\mathcal{C}$, we first note that the complementary cone field is contracted by $f^{-1}$. The intersection of the cones $\mathcal{C}^{n}(x)$ as in Lemma 2.6 defines at each point $x \in K$ a $d$-dimensional space $F(x)$. Considering the complementary cone field, we also obtain a $(\operatorname{dim}(M)-d)$-dimensional space $E(x)$ and, by the definition of contracted cones, the splitting $T_{x} M=E(x) \oplus F(x)$ is dominated. The second part of the lemma is obtained similarly.

2.2.3. Lift to Grassmannian bundles: the $r$-contracted case. In order to prove that an invariant submanifold is $C^{r}$, we will prove that its lift in a Grassmannian bundle is $C^{r-1}$. We explain here how to lift the dynamics. The 2-domination allows to get a domination of the lift dynamics.

Let us fix a contracted continuous cone field $\mathcal{C}$ of dimension $d$. Let $p: G(d, M) \rightarrow M$ be the Grassmannian bundle of $d$-dimensional tangent spaces. We define $\widehat{U}$, the interior of the set of $d$-dimensional tangent spaces $E$ contained in a cone $\mathcal{C}(x)$ for some $x \in U$. One gets a surjective submersion $p: \widehat{U} \rightarrow U$. By the second item of Definition 2.4, $D \Psi$ induces a continuous map

$$
\widehat{\Psi}: \widehat{U} \rightarrow G(d, M) .
$$

Note that $\widehat{\Psi}$ is $C^{r-1}$ if $\Psi$ is $C^{r}$. Moreover, $\widehat{\Psi}$ is a diffeomorphism if $\Psi$ is a $C^{2}$-diffeomorphism.

Proposition 2.11. If $\Psi$ is $C^{2}$, the map $\widehat{\Psi}$ contracts the fibers of $p: \widehat{U} \rightarrow U$. More precisely, there exists $C_{3}>0$ such that, for any $n \geqslant 1$, and for $P \in \widehat{U} \cap \cdots \cap \widehat{\Psi}^{-n+1}(\widehat{U})$, denoting $x=p(P)$,

$$
\left\|D \widehat{\Psi}(P)_{\mid p^{-1}(x)}\right\| \leqslant C_{3}\left\|D \Psi^{n}(x)_{\mid T_{x} M \backslash \mathcal{C}^{-n}(x)}\right\| m\left(D \Psi^{n}(x)_{\mid \mathcal{C}(x)}\right)^{-1}<C_{3} \lambda^{n} .
$$

Proof. For any two $d$-spaces $P, P^{\prime}$ in $T_{x} M$, one can consider the linear map $L: P \rightarrow$ $P^{\perp}$ whose graph is $P^{\prime}$. The tangent space at $P \in p^{-1}(x)$ to the fiber of $p$ may thus 
be identified to the space of linear maps $L: P \rightarrow P^{\perp}$ and $D \widehat{\Psi}$ acts by conjugacy. For $x \in U \cap \Psi^{-1}(U) \cap \cdots \cap \Psi^{-n+1}(U)$,

$$
D \widehat{\Psi}^{n}(L)=\Pi\left(P_{n}, P_{n}^{\perp}\right) \circ D \Psi^{n}(x) \circ L \circ D \Psi^{-n}\left(f^{n}(x)\right),
$$

where $\Pi(F, E)$ denotes the orthogonal projection on $E$ parallel to $F$ and $P_{n}=D \Psi^{n}(x)$. $P$. Let us consider a $(\operatorname{dim}(M)-d)$-dimensional space $E^{\prime}$ in $T_{\Psi^{n}(x)} M$ disjoint from $\mathcal{C}\left(\Psi^{n}(x)\right) \backslash\{0\}$ and its pre-image $E=D \Psi^{-n}\left(E^{\prime}\right)$. The projection between $E$ and $P^{\perp}$ parallel to $P$ is uniformly bounded and has a uniformly bounded inverse. The same holds for the projection between $D \Psi^{n}(x) \cdot E$ and $\left(D \Psi^{n}(x) \cdot P\right)^{\perp}$ parallel to $D \Psi^{n}(x) \cdot P$ (this is a consequence of Claim 2.7). One deduces that the norm of the linear map of $D \Psi^{n}(x)$ restricted to $E$ and the norm of $\Pi\left(P_{n}, P_{n}^{\perp}\right) \circ D \Psi^{n}(x)$ restricted to $P^{\perp}$ are equal up to a factor bounded by a uniform constant $C_{3}$ :

$$
\left\|D \widehat{\Psi}^{n}(P)\right\| \leqslant C_{3}\left\|D \Psi^{n}(x)_{\mid E}\right\| m\left(D \Psi^{n}(x)_{\mid P}\right)^{-1} .
$$

Together with the cone contraction, this concludes the proof.

Proposition 2.12. If $\Psi$ is $C^{2}$ and if $\mathcal{C}$ is $r$-contracted with $r \geqslant 2$, then there exists an $(r-1)$-contracted cone field $\widehat{\mathcal{C}}$ for $\widehat{\Psi}$, of dimension $d$, which is transverse to $p$. One can build $\widehat{\mathcal{C}}$ to contain any compact set of vectors $v \in T G(d, M)$ such that $D p \cdot v \in \mathcal{C} \backslash\{0\}$. If $\mathcal{C}$ is transverse to a submersion $\pi$, then one can build $\widehat{\mathcal{C}}$ to be transverse to $\pi \circ p$.

Proof. We define at each point $P \in G(d, M)$ the space $G(P)$ tangent to the fibers of $p$ and $H(P)$ a transverse space (for instance the normal space to $G(P)$ for an arbitrary Riemannian structure), so that $G, H$ are two smooth transverse bundles and $D p$ induces an isomorphism between the bundles $H$ and $T M$. One can thus pull back the Riemannian metric of $M$ as a metric $\|\cdot\|_{H}$ on $H$. Let us consider an arbitrary metric $\|\cdot\|_{G}$ on the bundle $G$, and define $\|\cdot\|=\left(\|\cdot\|_{H}^{2}+\varepsilon^{2}\|\cdot\|_{G}^{2}\right)^{1 / 2}$ a Riemannian metric on $G(d, M)$ for some $\varepsilon>0$ small. Note that for any vector $v$ at $P$ we have $\|D p \cdot v\| \leqslant\|v\|$, with equality if $v$ is tangent to $H(P)$.

We then define $\widehat{\mathcal{C}}(P)$ as the set of vectors $v$ at $P$ such that $\|D p \cdot v\| \geqslant \frac{1}{\sqrt{2}}\|v\|$ and $D p \cdot v \in \mathcal{C}(x)$ with $x=p(P)$. If $E$ is a $d$-dimensional subspace contained in $\mathcal{C}(x)$ and $F$ a $(\operatorname{dim}(T)-d)$-dimensional transverse subspace disjoint from $\mathcal{C}(x) \backslash\{0\}$, then $\widehat{\mathcal{C}}(P)$ contains $D p^{-1}(E) \cap H_{P}$ and is disjoint from $D p^{-1}(F) \backslash\{0\}$. Hence $\widehat{\mathcal{C}}$ is a continuous cone field of dimension $d$ transverse to $p$. If $\mathcal{C}$ is transverse to a submersion $\pi$, one may choose for $F$ the tangent space at $x$ of the fiber of $\pi$, which implies that $\widehat{\mathcal{C}}$ is transverse to the submersion $\pi \circ p$.

Let us choose $n_{0} \geqslant 1$ large enough. The small constant $\varepsilon>0$ will be fixed later. In order to prove that Definition 2.4 is satisfied, it will be enough to check it for any $n \in$ $\left\{n_{0}, \ldots, 2 n_{0}\right\}$. For any $P \in \widehat{U} \cap \cdots \cap \widehat{\Psi}^{-n+1}(\widehat{U})$, we set $x=p(P)$ and take any $v \in \widehat{\mathcal{C}}(P)$. By invariance of the cone field $\mathcal{C}$ we have $D p\left(\Psi^{n}(P)\right) \cdot\left(D \widehat{\psi}^{n} \cdot v\right) \in \mathcal{C}\left(\Psi^{n}(x)\right)$. One can decompose $v=v^{H}+v^{G}$ according to the splitting $H \oplus G$. By definition of $\widehat{\mathcal{C}}$, we have $v^{H} \in \mathcal{C}(x)$. By definition of the metric, we have $\left\|v^{H}\right\|_{H} \geqslant \varepsilon\left\|v^{G}\right\|_{G}$.

The image $w$ of $v^{H}$ by $D \widehat{\Psi}^{n}$ decomposes as $w^{H}+w^{G}$ with $\left\|w^{G}\right\|_{G} \leqslant K\left\|w^{H}\right\|_{H}$, where $K$ is a constant which controls the angle between the image $D \widehat{\Psi}^{n} \cdot E$ of the bundle $E$ and 
the fibers of $p$ for any $n_{0} \leqslant n \leqslant 2 n_{0}$. For $\varepsilon$ small, we get

$$
\left\|w^{H}\right\|_{H} \geqslant 2 \varepsilon\left\|w^{G}\right\|_{G}
$$

Since the metric on $H$ is defined by lifting the metric on $U$, we get

$$
\left\|w^{H}\right\|_{H} \geqslant m\left(D \Psi^{n}(x)_{\mid \mathcal{C}(x)}\right)\left\|v^{H}\right\|_{H} .
$$

By Proposition 2.11, we have

$$
C_{0}\left\|D \Psi^{n}(P)_{\mid T_{x} M \backslash \mathcal{C}^{-n}(x)}\right\| m\left(D \Psi^{n}(x)_{\mid \mathcal{C}(x)}\right)^{-1}\left\|v^{G}\right\|_{G} \geqslant\left\|D \widehat{\Psi}^{n}(P) \cdot v^{G}\right\|_{G} .
$$

Hence this gives

$$
m\left(D \Psi^{n}(x)_{\mid \mathcal{C}(x)}\right)^{-2}\left\|D \Psi^{n}(P)_{\mid T_{x} M \backslash \mathcal{C}^{-n}(x)}\right\|\left\|w^{H}\right\|_{H} \geqslant \frac{\varepsilon}{C_{0}}\left\|D \widehat{\Psi}^{n}(P) \cdot v^{G}\right\|_{G} .
$$

Since $\mathcal{C}$ is 2-contracted and $n$ is large, $m\left(D \Psi^{n}(x)_{\mid \mathcal{C}(x)}^{-2}\right)\left\|D \Psi^{n}(P)_{\mid T_{x} M \backslash \mathcal{C}^{-n}(x)}\right\|$ is small, and

$$
\left\|w^{H}\right\|_{H} \geqslant 2 \varepsilon\left\|D \widehat{\Psi}^{n}(P) \cdot v^{G}\right\|_{G} .
$$

With (2), one deduces $\left\|w^{H}\right\|_{H} \geqslant \frac{1}{2}\left\|D \widehat{\Psi}^{n}(P) \cdot v\right\|$, so that $D \widehat{\Psi}^{n}(P) \cdot v$ belongs to $\widehat{\mathcal{C}}\left(\Psi^{n}(P)\right)$. This gives the first item of Definition 2.4.

By the second item of Definition 2.4, and since $\left\|w^{H}\right\|_{H}=\left\|D \Psi^{n}(x) \cdot v^{H}\right\|_{H}$ does not vanish, the image $D \widehat{\Psi}^{n} \cdot v$ is non-zero. Hence the second item of Definition 2.4 is satisfied.

Let us fix two unit vectors $u \in \widehat{\mathcal{C}}(P), v \in T \widehat{U} \backslash \widehat{\mathcal{C}}^{-n}(P)$ and $n \in\left\{n_{0}, \ldots, 2 n_{0}\right\}$. We have

$$
\left\|D \widehat{\Psi}^{n}(P) \cdot u\right\| \geqslant\left\|D p\left(D \widehat{\Psi}^{n}(P) \cdot u\right)\right\|=\left\|D \Psi^{n}(x) \cdot(D p \cdot u)\right\| \geqslant \frac{1}{2} m\left(D \Psi^{n}(x)_{\mid \mathcal{C}(x)}\right) .
$$

For $D \widehat{\Psi}^{n} \cdot v$, two cases are possible. In the first case, $\left\|D p\left(D \widehat{\Psi}^{n} \cdot v\right)\right\| \leqslant \frac{1}{2}\left\|D \widehat{\Psi}^{n} \cdot v\right\|$. We decompose as $v=v^{H}+v^{G}$ and the image $w=D \widehat{\Psi}^{n} \cdot v^{H}$ as $w=w^{H}+w^{G}$. As before, we have $\left\|w^{G}\right\|_{G} \leqslant K\left\|w^{H}\right\|_{H}$. The first case restates as $\left\|w^{H}\right\|_{H} \leqslant \varepsilon\left\|w^{G}+D \widehat{\Psi}^{n} \cdot v^{G}\right\|_{G}$. Hence if $\varepsilon$ has been chosen small enough $\left\|w^{G}\right\|_{G}$ is much smaller than $\left\|D \widehat{\Psi}^{n} \cdot v^{G}\right\|_{G}$. With Proposition 2.11, one gets

$$
\begin{aligned}
\left\|D \widehat{\Psi}^{n} \cdot v\right\| & \leqslant 3 \varepsilon\left\|D \widehat{\Psi}_{\mid p^{-1}(x)}^{n}\right\|_{G}\left\|v^{G}\right\|_{G} \\
& \leqslant 3 C_{3}\left\|D \Psi^{n}(x)_{\mid T_{x} M \backslash \mathcal{C}^{-n}(x)}\right\| m\left(D \Psi^{n}(x)_{\mid \mathcal{C}(x)}\right)^{-1} \varepsilon\left\|v^{G}\right\|_{G} .
\end{aligned}
$$

Hence by the $r$-contraction of the cone field $\mathcal{C}$, and since $\varepsilon\left\|v^{G}\right\|_{G} \leqslant\|v\|=1$, this gives

$$
\frac{\left\|D \widehat{\Psi}^{n} \cdot v\right\|}{\min \left(\left\|D \widehat{\Psi}^{n} \cdot u\right\|,\left\|D \widehat{\Psi}^{n} \cdot u\right\|^{r-1}\right)} \leqslant \frac{6 \varepsilon C_{3}\left\|D \Psi^{n}(x)_{\mid T_{x} M \backslash \mathcal{C}^{-n}(x)}\right\|}{\min \left(m\left(D \Psi^{n}(x)_{\mid \mathcal{C}(x)}\right)^{2}, m\left(D \Psi^{n}(x)_{\mid \mathcal{C}(x)}\right)^{r}\right)} \leqslant 6 \varepsilon C_{3} \lambda^{-n} .
$$

In the other case, $\left.\| D p\left(D \widehat{\Psi}^{n} \cdot v\right)\right)\left\|\geqslant \frac{1}{2}\right\| D \widehat{\Psi}^{n} \cdot v \|$, and $D p\left(D \widehat{\Psi}^{n} \cdot v\right)=D \Psi^{n}(D p \cdot v)$ belongs to $T U \backslash \mathcal{C}$. By the cone contraction, one gets

$$
\begin{aligned}
\left\|D \widehat{\Psi}^{n} \cdot v\right\| & \left.\leqslant 2 \| D p\left(D \widehat{\Psi}^{n} \cdot v\right)\right)\|=2\| D \Psi^{n}(D p \cdot v) \| \\
& \leqslant 2 \lambda^{-n} \min \left(\left\|D \Psi^{n}(D p \cdot u)\right\|,\left\|D \Psi^{n}(D p \cdot u)\right\|^{r}\right) .
\end{aligned}
$$

We also have $\left\|D \Psi^{n}(D p \cdot u)\right\|=\left\|D p\left(D \widehat{\Psi}^{n} \cdot u\right)\right\| \leqslant\left\|D \widehat{\Psi}^{n} \cdot u\right\|$. Consequently,

$$
\frac{\left\|D \widehat{\Psi}^{n} \cdot v\right\|}{\min \left(\left\|D \widehat{\Psi}^{n} \cdot u\right\|,\left\|D \widehat{\Psi}^{n} \cdot u\right\|^{r}\right)} \leqslant 2 \lambda^{-n} .
$$

In both cases, the third item of Definition 2.4 holds; hence the cone $\widehat{\mathcal{C}}$ is $(r-1)$-contracted.

For $\varepsilon>0$ small enough, $\widehat{\mathcal{C}}$ contains any compact set of vectors $v$ satisfying $D p \cdot v \in$ $\mathcal{C} \backslash\{0\}$. 
2.2.4. Lifts to Grassmannian bundles: the bunched case. In Corollary 1.11, we will prove the existence of locally constant foliations. These are built from locally constant $C^{1}$-vector fields that are obtained as invariant sections of the tangent bundle. A different dominated splitting of the lift dynamics is used; it is a consequence of a bunching property.

Definition 2.13. The cone field $\mathcal{C}$ is bunched if there exist $\lambda>1$ and $n_{0} \geqslant 1$ such that, for any $n \geqslant n_{0}$, any $x$ in $U \cap \Psi^{-1}(U) \cap \cdots \cap \Psi^{-n+1}(U)$, and any unit vectors $u, v \in \mathcal{C}(x)$ and $w \in T_{x} M \backslash \mathcal{C}^{-n}(x)$, we have

$$
\left\|D \Psi^{n}(x) \cdot w\right\|<\lambda^{-n} \frac{\left\|D \Psi^{n}(x) \cdot u\right\|}{\left\|D \Psi^{n}(x) \cdot v\right\|} .
$$

Remark 2.14. When $\Psi$ is a diffeomorphism and $K$ is a partially hyperbolic invariant set such that $T M_{\mid K}=E^{s s} \oplus E^{c}$ and $\operatorname{dim}\left(E^{c}\right)=1$, then the cone fields $\mathcal{C}$ associated to $E^{c}$ as in (1) on a neighborhood of $K$ are bunched. Indeed, by Lemma 2.8, the vectors in the cone field $T M \backslash \mathcal{C}^{-n}$ are close to the bundle $E^{s s}$, and hence are contracted by forward iterations, while the vectors in the cone $\mathcal{C}$ become close to the bundle $E^{c}$ after a few iteration; since $E^{c}$ is one-dimensional, the iterates of any two vectors $u, v \in \mathcal{C}$ are almost collinear, and the ratio $\frac{\left\|D \Psi^{n}(x) \cdot u\right\|}{\left\|D \Psi^{n}(x) \cdot v\right\|}$ does not decay faster than the strong stable contraction.

Proposition 2.15. Let $\Psi$ be a $C^{2}$-diffeomorphism between $U$ and its image, and let $\mathcal{C}$ be a contracted cone field of dimension $d$. If the complementary cone field of $\mathcal{C}$ is bunched, then there exists a continuous cone field $\widehat{\mathcal{C}}$ of dimension $\operatorname{dim}(M)$ on $\widehat{U} \subset G(d, M)$ which is contracted by $\widehat{\Psi}$ and transverse to the submersion $p$.

Proof. With the same notation as in the proof of Proposition 2.12, we define in this case $\widehat{\mathcal{C}}(P)$ as the set of vectors $v$ at $P$ such that $\|D p \cdot v\| \geqslant \frac{1}{2}\|v\|$, and we obtain in this way a continuous cone field of $\operatorname{dimension} \operatorname{dim}(M)$ on $G(d, M)$, transverse to $p$. Since $\widehat{\Psi}$ is a local diffeomorphism, $D \widehat{\Psi}(x) \cdot u$ does not vanish on non-zero vectors. It is enough to prove the cone contraction for any integer $n \in\left\{n_{0}, \ldots, 2 n_{0}\right\}$, where $n_{0}$ is large.

Let us consider $v \in \widehat{\mathcal{C}}(P)$. It decomposes as $v=v^{H}+v^{G}$. One can decompose the image $w=D \widehat{\Psi}^{n} \cdot v^{H}$ as $w=w^{H}+w^{G}$. On the one hand, having chosen $\varepsilon$ small enough, one has

$$
2 \varepsilon\left\|v^{G}\right\|_{G} \leqslant\left\|v^{H}\right\|_{H} .
$$

On the other hand, by Lemma 2.8 ,

$\left\|w^{H}\right\|_{H} \geqslant m\left(D \Psi^{n}\right)\left\|v^{H}\right\|_{H} \geqslant m\left(D \Psi^{n}(x)\right) \varepsilon\left\|v^{G}\right\|_{G} \geqslant C_{2} l m\left(D \Psi^{n}(x)_{\mid T_{x} M \backslash \mathcal{C}^{-n}(x)}\right) \varepsilon\left\|v^{G}\right\|_{G}$.

By Proposition 2.11 and the bunching, we have

$$
\begin{aligned}
2 \varepsilon\left\|D \widehat{\Psi}^{n} \cdot v^{G}\right\|_{G} & \leqslant 2 \varepsilon C_{3}\left\|D \Psi^{n}(x)_{\mid T_{x} M \backslash \mathcal{C}^{-n}(x)}\right\| m\left(D \Psi^{n}(x)_{\mid \mathcal{C}(x)}\right)^{-1}\left\|v^{G}\right\|_{G} \\
& \leqslant 2 C_{3} C_{2}^{-1} \frac{\left\|D \Psi^{n}(x)_{\mid T_{x} M \backslash \mathcal{C}^{-n}(x)}\right\|}{m\left(D \Psi^{n}(x)_{\mid T_{x} M \backslash \mathcal{C}^{-n}(x)}\right) m\left(D \Psi^{n}(x)_{\mid \mathcal{C}(x)}\right)}\left\|w^{H}\right\|_{H} \leqslant\left\|w^{H}\right\|_{H} .
\end{aligned}
$$

Together with (3), this proves the first item of Definition 2.4. 
Let us fix two unit vectors $u \in \widehat{\mathcal{C}}(P), v \in T \widehat{U} \backslash \widehat{\mathcal{C}}^{-n}(P)$, and $n \in\left\{n_{0}, \ldots, 2 n_{0}\right\}$. Arguing as in the proof of Proposition 2.12, on the one hand we have

$$
\left\|D \widehat{\Psi}^{n}(P) \cdot u\right\| \geqslant \frac{1}{2} m\left(D \Psi^{n}(x)\right) \geqslant \frac{C_{2}}{2} m\left(D \Psi^{n}(x)_{\mid T_{x} M \backslash \mathcal{C}^{-n}(x)}\right) .
$$

On the other hand we have

$$
\left\|D \widehat{\Psi}^{n} \cdot v\right\| \leqslant 3 C_{3}\left\|D \Psi^{n}(x)_{\mid T_{x} M \backslash \mathcal{C}^{-n}(x)}\right\| m\left(D \Psi^{n}(x)_{\mid \mathcal{C}(x)}\right)^{-1} \varepsilon\left\|v^{G}\right\|_{G} .
$$

Hence, by the bunching of the cone field $\mathcal{C}$, and since $\varepsilon\left\|v^{G}\right\|_{G} \leqslant\|v\|$, this gives

$$
\frac{\left\|D \widehat{\Psi}^{n} \cdot v\right\|}{\left\|D \widehat{\Psi}^{n} \cdot u\right\|} \leqslant \frac{6 \varepsilon C_{3} C_{2}^{-1}\left\|D \Psi^{n}(x)_{\mid T_{x} M \backslash \mathcal{C}^{-n}(x)}\right\|}{m\left(D \Psi^{n}(x)_{\mid T_{x} M \backslash \mathcal{C}^{-n}(x)}\right) \cdot m\left(D \Psi^{n}(x)_{\mid \mathcal{C}(x)}\right)} \leqslant 6 \varepsilon C_{3} C_{2}^{-1} \lambda^{-n} .
$$

This gives the last item of Definition 2.4, and hence the contraction of the cone field $\mathcal{C}$.

\section{Existence of submanifolds carrying a compact set}

Let $K$ be a subset of the $n$-dimensional manifold $M$.

Definition 3.1. At each point $z \in K$ the tangent set $T_{z} K$ of $K$ is defined as follow.

For any chart $\varphi: U \rightarrow \mathbb{R}^{n}$ centered at $z$, and for $\varepsilon>0$, one considers the compact set

$$
\tau_{\varepsilon}=\text { Closure }\left\{v \in \mathbb{R}^{n}, \exists x, y \in \varphi(K) \cap B(z, \varepsilon), x \neq y \text { and } v=\frac{x-y}{\|x-y\|}\right\} .
$$

One denotes by $\tau_{0}$ the intersection $\bigcap_{\varepsilon>0} \tau_{\varepsilon}$, and by $T$ the linear subspace of $\mathbb{R}^{n}$ generated by $\tau_{0}$. The pull-back $T_{z} K:=\left(D_{z} \varphi\right)^{-1}(T)$ does not depend on the choice of the chart $\varphi$.

It is clear that a necessary condition for $K$ to be contained in a $d$-dimensional submanifold of $M$ is that each $T_{x} K$ is contained in a continuous subbundle of dimension $d$ of the restriction of $T M$ over $K$. The next theorem is an easy consequence of Whitney's extension theorem, and it asserts that this condition is also sufficient.

Theorem 3.1. If $K \subset M$ is a compact set and $x \mapsto E(x) \subset T_{x} M$ is a continuous $d$-dimensional subbundle defined on $K$, such that $T_{x} K \subset E(x)$ for any $x \in K$, then there is a compact $d$-dimensional $C^{1}$-submanifold with boundary $\Sigma \subset M$ which contains $K$ in its interior. Furthermore, $\Sigma$ is tangent to $E(x)$ at each point $x \in K$.

We now consider the case when $K$ is a partially hyperbolic set.

Corollary 3.2. Let $f$ be a $C^{1}$-diffeomorphism of $M$ and $K$ be a compact invariant set admitting a partially hyperbolic structure $T M_{\mid K}=E^{c} \oplus E^{u u}$, where $d=\operatorname{dim}\left(E^{c}\right)$. If, for each $x \in K$, the intersection $W^{u u}(x) \cap K$ is reduced to $\{x\}$, then there exists a compact d-dimensional $C^{1}$-submanifold with boundary which contains $K$ in its interior. Furthermore, at each $x \in K$, it is tangent to $E^{c}(x)$.

Remark 3.3. One can assume that $\Sigma \backslash K$ is smooth. Indeed, one can modify $\Sigma$ outside a small neighborhood $U$ of $K$ by an arbitrarily small $C^{1}$-perturbation, such that $\Sigma \backslash U$ is smooth. A converging sequence of such perturbation when $U$ decreases to $K$ gives the property. 


\subsection{Whitney's extension theorem: the solution of the local problem}

One can find in $[1$, Appendix A] the following statement.

Whitney's extension theorem. Let $A \subset \mathbb{R}^{d}$ be a closed subset, and let $f: A \rightarrow \mathbb{R}^{n-d}$ be a continuous function. The two following properties are equivalent.

(1) $f$ extends to a $C^{1}$ function $\Phi: \mathbb{R}^{d} \rightarrow \mathbb{R}^{n-d}$.

(2) There is a continuous map D from A to the space of linear maps $L\left(\mathbb{R}^{d}, \mathbb{R}^{n-d}\right)$ such that, if one defines the function $R: A \times A \rightarrow \mathbb{R}^{n-d}$ by

$$
R(x, y)=(f(y)-f(x))-D(x)(y-x),
$$

then for each $z \in A$ the quantity $\frac{\|R(x, y)\|}{\|y-x\|}$ tends to 0 as $x \neq y$ tend to $z$.

Moreover, if $f$ and $D$ verify the second property, then the extension $\Phi$ can be chosen so that $D_{x} \Phi=D(x)$ at each $x \in A$.

It can be restated as follows.

Corollary 3.4. Let $K \subset \mathbb{R}^{n}$ be a compact set such that

- every $(n-d)$-dimensional affine space $\left(x_{1}, \ldots, x_{d}\right) \times \mathbb{R}^{n-d}$ meets $K$ in at most one point,

- for every $x \in K$ there is a linear subspace $E(x) \subset \mathbb{R}^{n}$ of dimension $d$, transverse to $\{0\}^{d} \times \mathbb{R}^{n-d}$, containing the tangent set $T_{x} K$ of $K$ at $x$,

- the map $x \mapsto E(x)$ is continuous.

Then $K$ is contained in the graph $\Gamma$ of a $C^{1}-$ map $\Phi: \mathbb{R}^{d} \rightarrow \mathbb{R}^{n-d}$, and the tangent space $T_{x} \Gamma$ coincides with $E(x)$ at each point $x$ of $K$.

Proof. Let us denote by $A$ the projection of $K$ on $\mathbb{R}^{d} \times\{0\}^{n-d}$ along the vertical direction: $K$ is the graph of a function $f: A \rightarrow \mathbb{R}^{n-d}$. Since $K$ is compact, this map is continuous.

For each point $x$, the $d$-dimensional space $E(x)$ has been assumed to be transverse to the vertical direction so that it is the graph of a linear map $D(x): \mathbb{R}^{d} \rightarrow \mathbb{R}^{n-d}$. The map $x \mapsto D(x)$ is continuous since $x \mapsto E(x)$ is continuous.

Consider a point $z \in A$ and $p=(z, f(z))$ in $K$. The hypothesis that $T_{p} K \subset E(p)$ means that every $v \in T_{p} K$ can be written as $(u, D(z) u)$. Hence, for any $x, y \in A$ in a small neighborhood of $z$, the following quantity is very small:

$$
\frac{(x-y, f(x)-f(y))}{\|(x-y, f(x)-f(y))\|}-\frac{(x-y, D(z)(x-y))}{\|(x-y, D(z)(x)-y)\|} .
$$

After multiplying by the uniformly bounded quotient $\frac{\|(x-y, D(z)(x-y))\|}{\|x-y\|}$, we get that

$$
\frac{\|(x-y, D(z)(x-y))\|}{\|(x-y, f(x)-f(y))\|} \frac{(x-y, f(x)-f(y))}{\|x-y\|}-\frac{(x-y, D(z)(x-y))}{\|x-y\|} \rightarrow 0
$$

when $x$ and $y \neq x$ tend to $z$.

Considering the projection on the horizontal coordinates $\mathbb{R}^{d}$, one deduces that $\frac{\|(x-y, D(z)(x-y))\|}{\|(x-y, f(x)-f(y))\|}$ goes to 1 . Since $\frac{\|(x-y, D(z)(x-y))\|}{\|x-y\|}$ is uniformly bounded, we deduce 
from (4) that

$$
\frac{(x-y, f(x)-f(y))}{\|x-y\|}-\frac{(x-y, D(z)(x-y))}{\|x-y\|} \rightarrow 0 \quad \text { when } x \neq y \text { tend to } z .
$$

By projecting on the vertical coordinates $\mathbb{R}^{n-d}$, and by continuity of $x \mapsto D(x)$ at $z$, one gets

$$
\frac{f(x)-f(y)-D(x)(x-y)}{\|x-y\|} \rightarrow 0 \quad \text { when } x \neq y \text { tend to } z .
$$

This gives the second of the properties of Whitney's extension theorem, and this theorem concludes the proof of the corollary.

Consider now a subset $K$ of the $n$-dimensional manifold $M$ and a $d$-dimensional linear subspace $E(x) \subset T_{x} M$ at each point $x \in K$. We introduce two definitions.

- A diffeomorphism $\varphi$ from an open set $U \subset M$ to $]-1,1\left[{ }^{n}\right.$ is called an adapted chart of $(K, E)$ if $\varphi(K \cap U) \subset]-1,1\left[^{d} \times\{0\}^{n-d}\right.$ and $D \varphi(x) \cdot E(x)$ coincides with the linear space $\mathbb{R}^{d} \times\{0\}^{n-d}$ for each $x \in K \cap U$.

- A pair $(U, \Sigma)$, where $U \subset M$ is open and $\Sigma \subset U$ is a submanifold, carries $(K, E)$ if $K \cap U \subset \Sigma$ and $T_{x} \Sigma=E(x)$ for each $x \in K \cap U$.

Corollary 3.5. Let $K \subset M$ be compact. If the map $x \mapsto E(x)$ is continuous on $K$ and satisfies $T_{x} K \subset E(x)$ for each $x \in K$, then each point of $K$ is contained in an adapted chart of $(K, E)$.

Proof. Let us choose some coordinates around a point $p \in K$ such that the vertical plane $\{0\}^{d} \times \mathbb{R}^{n-d}$ is transverse at $p$ to $E(p)$. As $T_{p} K \subset E(p)$, shrinking the chart at $p$ if necessary, one can assume that, for $x \neq y$ in $K$ close to $p, \frac{x-y}{\|x-y\|}$ does not belong to the vertical $(n-d)$-dimensional plane. Hence, any vertical $(n-d)$-dimensional affine space in this chart meets $K$ in at most one point. We can thus apply Corollary 3.4 and get a $C^{1}$-graph $\Gamma$. A chart at $p$ which trivializes the graph is an adapted chart of $(K, E)$.

\subsection{From local to global}

Theorem 3.1 is now a consequence of Corollary 3.5 and of the following proposition.

Proposition 3.6. Let $K \subset M$ be a compact subset, $d>0$ be an integer, and at each $x \in K$ let $E(x) \subset T_{x} M$ be a d-dimensional subspace such that $K$ is covered by charts adapted to $(K, E)$.

Then there exists an open d-dimensional submanifold $\Sigma \subset M$ such that $K \subset \Sigma$ and $T_{x} \Sigma=E(x)$ for $x \in K$.

Proof. Consider a finite covering $\left\{U_{i}\right\}_{i \in\{1, \ldots, \ell\}}$ of $K$ by charts adapted to $(K, E)$, and for each $i$ fix some open subset $V_{i}$ whose closure is contained in $U_{i}$, such that $K \subset \bigcup_{i} V_{i}$. By induction, one will build open sets $W_{i}$, for $i=1, \ldots, \ell$, containing $O_{i}:=\overline{\bigcup_{j=1}^{i} V_{j}}$ and a submanifold $\Sigma_{i}$ such that the pair $\left(W_{i}, \Sigma_{i}\right)$ carries $(K, E)$. The open submanifold $\Sigma_{\ell}$ obtained this way will satisfy the conclusion of Proposition 3.6 since $\left(W_{\ell}, \Sigma_{\ell}\right)$ carries $(K, E)$ and since $K$ is contained in $W_{\ell}$. 
For the first step of the induction, one chooses $W_{1}=U_{1}$, and $\Sigma_{1}$ is the horizontal $d$-dimensional plane in the coordinates of $U_{1}$. The other steps are obtained by applying the next lemma, Lemma 3.7, to $U_{i+1}, V_{i+1}, W_{i}, \Sigma_{i}$ and $O_{i}$.

Lemma 3.7. Let $U$ be an adapted chart of $(K, E)$, and $(W, \Sigma)$ be a pair carrying $(K, E)$. Consider an open set $V$ whose closure is contained in $U$ and an open set $O$ whose closure is contained in $W$. Then there is a pair $\left(W^{\prime}, \Sigma^{\prime}\right)$ carrying $(K, E)$ such that $\overline{V \cup O} \subset W^{\prime}$.

The proof of Lemma 3.7 is obtained after two intermediate lemmas.

In the first lemma, we prove that, in the coordinates of the adapted chart $U, \Sigma$ can be considered as a graph. We recall that the adapted chart identifies $U$ with $]-1,1{ }^{n}$ and we introduce some constants.

$-\eta<\delta$ in ]0, 1[ are chosen to define the smaller rectangles ]- $\delta, \delta\left[{ }^{n},\right]-\eta, \eta{ }^{n}$ of $U$. By choosing $\eta$ close to 1 , one can assume that they contain $\bar{V}$.

$-\varepsilon>0$ allows us to define the (open) $\varepsilon$-neighborhood $W_{\varepsilon}$ of $\bar{O}$ and the intersection $\Sigma_{\varepsilon}:=\Sigma \cap W_{\varepsilon}$. Notice that the pair $\left(W_{\varepsilon}, \Sigma_{\varepsilon}\right)$ still carries $(K, E)$.

Sublemma 3.8. For $\varepsilon>0$ small enough, the intersection $\left.\Sigma_{\varepsilon} \cap\right]-\delta, \delta\left[{ }^{n}\right.$ is the graph of a $C^{1}$-function $\left.\Phi: S \rightarrow\right]-\eta, \eta\left[{ }^{n-d}\right.$ defined on an open subset of $]-\delta, \delta\left[{ }^{d}\right.$. Furthermore, if $z \in S \times]-\delta, \delta\left[{ }^{n-d}\right.$ belongs to $K$, then $z$ belongs to the graph of $\Phi$.

Proof. Consider a point $z=(x, 0) \in]-1,1\left[{ }^{d} \times\{0\}^{n-d}\right.$.

- Assume first that $z \notin K \cap \bar{O}$. Then the fiber $\{x\} \times[-\delta, \delta]^{n-d}$ is a compact set disjoint from $K \cap \bar{O}$. For $\varepsilon>0$ small enough, $\Sigma_{\varepsilon}$ is contained in an arbitrarily small neighborhood of $K \cap \bar{O}$. As a consequence, there is an open neighborhood $S_{z}$ of $z$ in ]$-1,1\left[{ }^{d} \times\{0\}^{n-d}\right.$ and there is a number $\varepsilon(z)>0$ such that $\left(S_{z} \times[-\delta, \delta]^{n-d}\right) \cap \Sigma_{\varepsilon(z)}$ is empty.

- Assume now that $z \in K \cap \bar{O}$. The submanifold $\Sigma$ is tangent at $z$ to $]-1,1\left[^{d}\right.$ $\times\{0\}^{n-d}$; as a consequence, there is an open neighborhood $V_{z}$ of $z$, contained in (]$-1,1\left[{ }^{d} \times\right]-\eta, \eta\left[{ }^{n-d}\right) \cap W$, such that the intersection of $\Sigma$ with $V_{z}$ is a graph over $V_{z} \cap(]-1,1\left[{ }^{d} \times\{0\}^{n-d}\right)$. Notice that the difference $\left(\{x\} \times[-\delta, \delta]^{n-d}\right) \backslash V_{z}$ is a compact set disjoint from $K$ (as $U$ is an adapted chart). Hence there is $\varepsilon(z)>0$, and there is an open neighborhood $V_{z}^{\prime}$ of $\left(\{x\} \times[-\delta, \delta]^{n-d}\right) \backslash V_{z}$ such that $V_{z}^{\prime}$ is disjoint from $\Sigma_{\varepsilon(z)}$ and $K$. One chooses an open neighborhood $\tilde{S}_{z}$ of $z$ in $]-1,1\left[{ }^{d} \times\{0\}^{n-d}\right.$ small enough such that $\tilde{S}_{z} \times[-\delta, \delta]^{n-d}$ is contained in the neighborhood $V_{z} \cup V_{z}^{\prime}$ of $\{x\} \times[-\delta, \delta]^{n-d}$.

By construction, $\left(\tilde{S}_{z} \times[-\delta, \delta]^{n-d}\right) \cap \Sigma_{\varepsilon(z)}$ is the graph of a $C^{1}$-function, defined over an open neighborhood $S_{z}$ of $z$ in $]-1,1\left[{ }^{d} \times\{0\}^{n-d}\right.$ and with values in $]-\eta, \eta\left[{ }^{n-d}\right.$.

Moreover, if $y \in K \cap\left(S_{z} \times[-\delta, \delta]^{n-d}\right)$ then $y$ belongs to $V_{z}$, and hence to $W$. In particular, $y \in \Sigma$; hence $y$ belongs to the graph of the function above, and in particular $y \in \Sigma_{\varepsilon(z)}$.

The constructions above associates to each point $z \in]-1,1\left[{ }^{d} \times\{0\}^{n-d}\right.$ an open neighborhood $S_{z}$ (in $]-1,1\left[{ }^{d} \times\{0\}^{n-d}\right.$ ) and a constant $\varepsilon(z)$. By compactness of $[-\delta, \delta]^{d}$, one can choose a finite set $X$ such that the open sets $S_{z}, z \in X$, cover $[-\delta, \delta]^{d}$. One fixes 
$\varepsilon>0$ less than $\varepsilon(z), z \in X$. Then $\left.\Sigma_{\varepsilon} \cap\right]-\delta, \delta\left[{ }^{n}\right.$ is the graph of a $C^{1}$-map $\Phi$ from an open subset $S \subset]-\delta, \delta\left[^{d}\right.$ to $]-\eta, \eta\left[{ }^{n-d}\right.$.

Finally, let $y$ be a point in $K \cap(S \times]-\delta, \delta\left[{ }^{n-d}\right)$. Then $y$ belongs to some $S_{z} \times[-\delta, \delta]^{n-d}$, $z \in X \cap K \cap \bar{O}$ and we have seen that $y \in \Sigma_{\varepsilon(z)}$; hence $y$ belongs to the graph of $\Phi$.

Let $S$ be the open set given by the previous lemma, and let $T$ be the open set $S \times$ ]$\delta, \delta\left[{ }^{n-d}\right.$. We have proved that $\left(W_{\varepsilon} \cup T, \Sigma_{\varepsilon}\right)$ carries $(K, E)$. We now modify $\Sigma_{\varepsilon}$ in the chart $U$ in order to glue it to the horizontal rectangle $]-\eta, \eta\left[{ }^{d} \times\{0\}^{n-d}\right.$. We thus choose a smooth function $\theta:]-1,1^{d} \rightarrow[0,1]$ which takes the value 0 in a neighborhood of $[-\eta, \eta]^{d}$ and takes the value 1 in a neighborhood of $]-1,1\left[^{d} \backslash\right]-\delta, \delta\left[^{d}\right.$.

Sublemma 3.9. There is an open submanifold $\Sigma_{\varepsilon}^{\prime}$ which coincides with $\Sigma_{\varepsilon}$ on $\left.M \backslash\right]-\delta, \delta\left[^{n}\right.$ and with the graph of $\theta \cdot \Phi: S \rightarrow]-\eta, \eta\left[{ }^{n-d}\right.$ in $]-\delta, \delta\left[{ }^{n}\right.$. Moreover, $\left(W_{\varepsilon} \cup T, \Sigma_{\varepsilon}^{\prime}\right)$ carries $(K, E)$.

Proof. Let us consider the set $\Sigma_{\varepsilon}^{\prime}$ union of $\left.\Sigma_{\varepsilon} \backslash\right]-\delta, \delta\left[{ }^{n}\right.$ with the graph of $\theta \cdot \Phi$ (contained in $S \times]-\eta, \eta\left[{ }^{n-d}\right)$. We will cover $M$ by two open sets and verify that the intersection of $\Sigma_{\varepsilon}^{\prime}$ with each of them is a submanifold of dimension $d$.

- In $M \backslash\left[-\delta^{\prime}, \delta^{\prime}\right]^{n}$, where $\delta^{\prime}$ is close to $\delta$, the sets $\Sigma_{\varepsilon}^{\prime}$ and $\Sigma_{\varepsilon}$ coincide. Indeed, by Sublemma 3.8, neither $\Sigma_{\varepsilon}^{\prime}$, nor $\Sigma_{\varepsilon}$ meets $[-\delta, \delta]^{d} \times(]-\delta, \delta\left[{ }^{n-d} \backslash\right]-\eta, \eta\left[{ }^{n-d}\right)$. Moreover, taking $\delta^{\prime}<\delta$ such that $\varphi$ is equal to 1 on $]-\delta, \delta\left[{ }^{d} \backslash\right]-\delta^{\prime}, \delta^{\prime}\left[{ }^{d}\right.$, one deduces that $\Sigma_{\varepsilon}^{\prime}$ and $\Sigma_{\varepsilon}$ coincide on (]$\left.-\delta, \delta\left[{ }^{d} \backslash\left[-\delta^{\prime}, \delta^{\prime}\right]^{d}\right) \times\right]-\delta, \delta\left[{ }^{n-d}\right.$.

- In $]-\delta, \delta\left[^{n}\right.$, the set $\Sigma_{\varepsilon}^{\prime}$ is the graph of a $C^{1}$-function defined on an open set of ]$-\delta, \delta\left[{ }^{d}\right.$.

This implies that $\Sigma_{\varepsilon}^{\prime}$ is a $d$-dimensional submanifold of $M$, which is contained in $W_{\varepsilon} \cup T$ (since $W_{\varepsilon}$ contains $\Sigma_{\varepsilon}$ and $T$ contains the graph of $\theta \cdot \Phi$ ).

Consider any $z \in K \cap\left(W_{\varepsilon} \cup T\right)$. If $z \in T$, then Sublemma 3.8 implies that $z$ coincides with $(x, \Phi(x))$ for some $x \in S$. But $\Phi(x)=0$ when $x \in K$ so that $\theta(x) . \Phi(x)=\Phi(x)=0$ and $z$ belongs to the graph of $\theta \Phi$ and hence to $\Sigma_{\varepsilon}^{\prime}$. If $z \in W_{\varepsilon} \backslash T$, then $z$ belongs to $\left.W_{\varepsilon} \backslash\right]-\delta, \delta\left[{ }^{n}\right.$ (the points of $\left.K \cap\right]-\delta, \delta\left[{ }^{n} \cap W_{\varepsilon}\right.$ belong to $]-\delta, \delta\left[{ }^{n} \cap \Sigma_{\varepsilon}\right.$ and hence in $T$, by Sublemma 3.8); in particular $x$ belongs to $\left.\Sigma_{\varepsilon} \backslash\right]-\delta, \delta\left[{ }^{n}\right.$, and to $\Sigma_{\varepsilon}^{\prime}$.

End of the proof of the Lemma 3.7. By Sublemma 3.9 , the union $\Sigma^{\prime}=\Sigma_{\varepsilon}^{\prime} \cup(]-$ $\eta, \eta\left[{ }^{d} \times\{0\}^{n-d}\right)$ is an open $C^{1}$-submanifold of dimension $d$. Let $W^{\prime}$ be the open set $\left.W_{\varepsilon} \cup T \cup\right]-\eta, \eta\left[{ }^{n}\right.$. Notice that $\bar{O} \subset W_{\varepsilon} \subset W^{\prime}$ and $\left.\bar{V} \subset\right]-\eta, \eta\left[{ }^{n} \subset W^{\prime}\right.$.

One concludes the proof by showing that $\left(W^{\prime}, \Sigma^{\prime}\right)$ carries $(K, E)$. If $x \in K \cap W^{\prime}$, then one of the following holds.

$-x \in]-\eta, \eta\left[^{n}\right.$. Then $\left.x \in\right]-\eta, \eta\left[{ }^{d} \times\{0\}^{n-d}\right.$, and since $U$ is a adapted chart of $(K, E)$, the space $E(x)$ is tangent to $]-\eta, \eta\left[{ }^{d} \times\{0\}^{n-d}\right.$. So $x \in \Sigma^{\prime}$, and $E(x)$ is tangent to $\Sigma^{\prime}$.

$-x \in W_{\varepsilon} \cup T$. Then $x \in \Sigma_{\varepsilon}^{\prime} \subset \Sigma^{\prime}$, and $E(x)$ is tangent to $\Sigma_{\varepsilon}^{\prime}$ (and to $\Sigma^{\prime}$ ) by Sublemma 3.9.

Proposition 3.6 and Theorem 3.1 are now proved. 


\subsection{Application to partially hyperbolic sets without strong connections}

By Theorem 3.1, Corollary 3.2 is a direct consequence of the following.

Proposition 3.10. Under the assumptions of Corollary 3.2, $T_{x} K \subset E^{c}(x)$ at each point $x \in K$.

As in $\S 2.2 .2$, one considers the cone fields $\mathcal{C}_{\beta}$ associated to a continuous extension of the bundles $E^{c}, E^{u u}$. To prove the proposition, it is enough to replace $f$ by an iterate $f^{k}$; hence one can assume the following properties for any $x$ close to $K$ :

$$
D f\left(\mathcal{C}_{1}(x)\right) \subset \mathcal{C}_{\frac{1}{2}}(f(x)),
$$

$2\|D f(u)\| \leqslant\|D f(v)\|$ and $\|D f(v)\| \geqslant 3$ for any unitary $u \in E(x), v \in \mathcal{C}_{1}(x)$.

For $\varepsilon>0$ less that the radius of the injectivity of the exponential map associated to the metric on the manifold $M$ (if $M$ is not compact, it suffices to consider a compact neighborhood of $K$ ), and given any two points $x, y \in M$ with $d(x, y)<\varepsilon$, there is a unique geodesic of length less than $\varepsilon$ joining $x$ to $y$. We will denote by $[x, y]$ this geodesic. If $\varepsilon$ is small and if $V \subset U$ is a small neighborhood of $K$, for any two points $x, y \in V$ with $d(x, y)<\varepsilon$, one has $[x, y] \subset U$.

Definition 3.11. The pair $(x, y)$ is in the cone field $\mathcal{C}_{1}$ if the tangent vector of the geodesic segment $[x, y]$ at each point $z \in[x, y]$ belongs to the cone $\mathcal{C}_{1}$.

The pairs of points contained in a same strong unstable leaf can be characterized as follows.

Lemma 3.12. Let $x, y \in K$ and $m \in \mathbb{N}$ such that for every $n \geqslant m$ one has $d\left(f^{-n}(x), d\left(f^{-n}(y)\right) \leqslant \varepsilon\right.$ and the pair $\left(f^{-n}(x), f^{-n}(y)\right)$ is in the cone field $\mathcal{C}_{1}$.

Then $x$ and $y$ belong to the same strong unstable manifold.

Proof. From the definition of partial hyperbolicity, for each $n \geqslant 0$ and each unitary $u \in$ $E^{c}(x)$,

$$
\left\|D f^{-n} \cdot u\right\| \geqslant 2^{n} d\left(f^{-n}(x), f^{-n}(y)\right) .
$$

This implies that $y$ belongs to the strong unstable manifold of $x$ (see [12, Theorem 5.1]).

The property for a pair of points of $K$ to be in the cone field $\mathcal{C}_{1}$ is invariant by positive iterates, as long as the distance between $x$ and $y$ remains small.

Lemma 3.13. There is $\delta \in] 0$, $\varepsilon$ [ such that, for any pair $(x, y)$ in $\mathcal{C}_{1}$ with $x, y \in K$ and $d(x, y)<\delta$,

$-d(f(x), f(y))<\varepsilon$,

- the pair $(f(x), f(y))$ is in the cone field $\mathcal{C}_{1}$,

$-d(f(x), f(y)) \geqslant 2 d(x, y)$. 
Proof. The first point follows from uniform continuity and the two others from the following properties.

- If $d(x, y)$ is small, the image $f([x, y])$ is $C^{1}$-close to the geodesic segment $[f(x), f(y)]$. In particular, the ratio of their length is arbitrarily close to 1 .

- For a pair $(x, y)$ in $\mathcal{C}_{1}$, the length of $f([x, y])$ is greater than 3 times $d(x, y)$ (by $\left.(1)\right)$.

- For a pair $(x, y)$ in $\mathcal{C}_{1}$, the segment $f([x, y])$ is tangent at each point to the cone field $\mathcal{C}_{\frac{1}{2}}$.

Proof of Proposition 3.10. Assume that there is some point $z \in K$ such that $T_{z} K \neq$ $E^{c}(z)$ : there exist $v \in T_{z} K \backslash E^{c}(z)$ and two sequences $\left(x_{n}\right),\left(y_{n}\right)$ in $K$ converging to $z$ such that in any chart at $z$ the vectors $\frac{y_{n}-x_{n}}{\left\|y_{n}-x_{n}\right\|}$ converge to $v$. Note that by replacing $v$ by an iterate $D f^{\ell} \cdot v /\left\|D f^{\ell} \cdot v\right\|$ one can assume $v \in \mathcal{C}_{1}(z)$, and hence each pair $\left(x_{n}, y_{n}\right)$ is in the cone field $\mathcal{C}_{1}$.

For large $n$, the distance $d\left(x_{n}, y_{n}\right)$ is arbitrarily small. Lemma 3.13 implies that there is $k_{n}>0$ such that $d\left(f^{k_{n}}\left(x_{n}\right), f^{k_{n}}\left(y_{n}\right)\right) \in\left[\frac{\delta}{\|D f\|}, \delta\right]$, and such that $d\left(f^{k}\left(x_{n}\right), f^{k}\left(y_{n}\right)\right)<\frac{\delta}{\|D f\|}$ for any $k \in\left\{0, \ldots, k_{n}-1\right\}$. By taking a subsequence, one can assume that the pairs $\left(f^{k_{n}}\left(x_{n}\right), f^{k_{n}}\left(y_{n}\right)\right)$ converge to a pair $(x, y)$ of points of $K$. We will prove now that $y \in W^{u u}(x)$.

For any $i>0$, the pair $\left(f^{-i}(x), f^{-i}(y)\right)$ is limit of the pairs $\left(f^{k_{n}-i}\left(x_{n}\right), f^{k_{n}-i}\left(y_{n}\right)\right)$. By the choice of $k_{n}$, the distances $\left.d\left(f^{k_{n}-i}\left(x_{n}\right), f^{k_{n}-i}\left(y_{n}\right)\right)\right)$ are less than $\delta$, so that $d\left(f^{-i}(x), f^{-i}(y)\right)<\varepsilon$. Applying Lemma 3.13 inductively, one gets that the pair $\left.d\left(f^{k_{n}-i}\left(x_{n}\right), f^{k_{n}-i}\left(y_{n}\right)\right)\right)$ is in the cone field $\mathcal{C}_{1}$. Notice that the geodesic segment $\left[f^{\left(k_{n}-i\right)}\left(x_{n}\right), f^{\left(k_{n}-i\right)}\left(y_{n}\right)\right]$ converges (in the $C^{1}$-topology) to the geodesic segment $\left[f^{-i}(x), f^{-i}(y)\right]$. As a consequence, the pair $\left(f^{-i}(x), f^{-i}(y)\right)$ is in the cone $\mathcal{C}_{1}$.

Lemma 3.12 now concludes that $x$ and $y$ belong to the same strong unstable manifold. Notice that $x \neq y$ because $x$ and $y$ are joined by a geodesic segment with length in $] 0, \varepsilon[$. This contradicts the hypotheses on $K$ in the statement of Corollary 3.2.

\section{Invariant center manifold}

We explain here how to replace the submanifold given by Theorem 3.1 by an invariant submanifold.

Theorem 4.1. Let $f$ be a diffeomorphism of a manifold $M$, and let $K$ be an invariant compact set contained in the interior of an (a priori non-invariant) compact submanifold with boundary $\Sigma$. One assumes furthermore that $K$ admits a partially hyperbolic splitting $T M=E^{c} \oplus E^{u u}$ such that $E^{c}(x)=T_{x} \Sigma$ at each point $x \in K$.

Then, one can replace $\Sigma$ by a submanifold $S$ which is locally invariant: $S \cap f(S)$ contains a neighborhood of $K$ in $S$.

The proof follows the usual construction of center manifolds for fixed points: one considers graphs of functions $h: \Sigma \rightarrow \mathbb{R}^{n-d}$, where $\mathbb{R}^{n-d}$ is a local coordinate transverse to $\Sigma$. The action of $f^{-1}$ can be modified outside a neighborhood of $K$ so that it preserves the space of Lipschitz graphs. The domination between $E^{c}$ and $E^{u u}$ ensures that this action is a contraction, and hence has a fixed point: this is the center manifold. 
In our setting, the fixed point has been replaced by an invariant compact set $K$ which makes the argument more delicate: the action of $f^{-1}$ in a neighborhood of $K$ cannot be approximated by a linear map, and the local transverse coordinates are obtained by the construction of a tubular neighborhood.

\subsection{First constructions}

In this section, we build a tubular neighborhood of the submanifold $\Sigma$ which will allow us to define the space of graphs.

4.1.1. Bundles $E, F$ around $K$. Since the splitting $T M_{\mid K}=E^{c} \oplus E^{u u}$ is partially hyperbolic, there exist $\lambda_{K}>1$ and $n_{K} \geqslant 1$ such that, for each $x \in K$ each $n \geqslant n_{K}$ and each unit vector $u \in E^{c}(x), v \in E^{u u}(x)$, one has

$$
\left\|D f^{n}(x) \cdot v\right\|>\lambda_{K}^{n}\left\|D f^{n}(x) \cdot u\right\| \quad \text { and } \quad\left\|D f^{n}(x) \cdot v\right\|>\lambda_{K}^{n} .
$$

From now on we assume that $n_{K}=1$ (this is always possible by changing the initial metric; see [10]).

We also extend the bundles $E^{c}, E^{u u}$, defined on $K$ as two (a priori non-invariant) continuous bundles $E, F$ on a neighborhood of $K$. One can reduce $\Sigma$ and assume that at each $x \in \Sigma E(x), F(x)$ are defined and that both $E(x)$ and $T_{x} \Sigma$ are transverse to $F(x)$.

4.1.2. Tubular neighborhoods of $\Sigma$. The next proposition provides us with a tubular neighborhood $T$ of an open submanifold $\Sigma_{0} \subset \Sigma$.

Definition 4.1. A tubular neighborhood of an open submanifold $\Sigma_{0} \subset \Sigma$ is a smooth surjective submersion $\pi: T \rightarrow \Sigma_{0}$ on an open neighborhood $T$ of $\Sigma_{0}$ which induces the identity on $\Sigma_{0}$.

A vector $u \in T_{x} M$ for $x \in T$ is vertical if it is tangent to the fibers of $\pi$ (i.e., $D \pi(x) . u=$ 0 ) and horizontal if it is tangent to $E$. The set $V_{x}$ of vertical vectors at $x$ is the vertical space. Any tangent vector $u \in T_{x} M$ decomposes as a sum $u_{v}+u_{h}$, where $u_{v}$ is vertical and $u_{h}$ is horizontal. For any $\beta>0$ we denote by $\mathcal{C}_{\beta}^{h}(x)$ the horizontal cone associated to the splitting $E \oplus V$ :

$$
\mathcal{C}_{\beta}^{h}(x)=\left\{u \in T_{x} M, \beta\left\|u_{h}\right\| \geqslant\left\|u_{v}\right\|\right\}
$$

Proposition 4.2. For any $\left.\lambda_{0} \in\right] 1, \lambda_{K}\left[\right.$ and any $\eta, \beta, \delta>0$ there is a neighborhood $\Sigma_{0}$ of $K$ in $\Sigma$ and there is a tubular neighborhood $\pi: T \rightarrow \Sigma_{0}$ with the following properties.

(1) For any vertical vector $u_{v}$ at $x \in T \cap f^{-1}(T)$,

$$
\left\|D f(x) \cdot u_{v}\right\| \geqslant \lambda_{0}\left\|u_{v}\right\| \quad \text { and } \quad\left\|D \pi \circ D f(x) \cdot u_{v}\right\| \leqslant \eta\left\|u_{v}\right\| .
$$

(2) For any horizontal vector $u_{h}$ at $x \in T$,

$$
(1-\delta)\left\|u_{h}\right\| \leqslant\left\|D \pi(x) \cdot u_{h}\right\| \leqslant(1+\delta)\left\|u_{h}\right\| .
$$

(3) For any $x \in T \cap f(T)$, one has

$$
D f^{-1}(x) \cdot \mathcal{C}_{\beta}^{h}(x) \subset \mathcal{C}_{\frac{\beta}{\lambda_{0}}}^{h}\left(f^{-1}(x)\right) .
$$


Proof. Let $\widetilde{F}$ be a smooth vector bundle arbitrarily $C^{0}$-close to $F$, let $\Sigma_{0}$ be a small open neighborhood of $K$ in $\Sigma$, and let $m_{0}>0$ be a small constant. We denote by $\mathcal{T}_{m_{0}}$ the $m_{0}$-neighborhood of the zero section of the restriction $\widetilde{F}_{\mid \Sigma_{0}}$. The exponential map sends $\mathcal{T}_{m_{0}}$ diffeomorphically on a set $T \subset M$. The canonical projection $\mathcal{T}_{m_{0}} \subset \widetilde{F}_{\mid \Sigma_{0}}$ onto $\Sigma_{0}$ induces by identification a projection $\pi: T \rightarrow \Sigma_{0}$. One checks easily that $T$ is a tubular neighborhood of $\Sigma_{0}$.

At points $x$ of $K$ the bundle $E^{u u}$ is preserved, expanded by $D f$ by $\lambda_{K}$, and the cone $\mathcal{C}_{\beta}^{h}(x)$ is mapped into $\mathcal{C}_{\beta / \lambda_{K}}^{h}(f(x))$. Since the splitting $E \oplus \widetilde{F}$ is close to the splitting $E^{c} \oplus E^{u u}$, the three items of the proposition follow by continuity.

As a consequence we obtain the following.

Lemma 4.3. Under the setting of Proposition 4.2, and if $\beta<\frac{\lambda_{0}-2 \eta}{6 \beta}$, for any vertical vector $u_{v} \neq 0$ at $x \in T \cap f^{-1}(T)$, the image $w=D f(x) \cdot u_{v}$ does not intersect the cone $\mathcal{C}_{3 \beta}^{h}(f(x))$.

Proof. One first decomposes $w$ into $w_{h}+w_{v}$ and denotes by $w_{\pi}=D \pi(x) \cdot w_{h}=D \pi(x) \cdot w$ the projection by $D \pi$. We have $\|w\| \geqslant \lambda_{0}\left\|u_{v}\right\|$ and $\left\|w_{\pi}\right\| \leqslant \eta\left\|u_{v}\right\|$ by Proposition 4.2(1). Moreover, $\left\|w_{\pi}\right\| \geqslant \frac{1}{2}\left\|w_{h}\right\|$ by Proposition $4.2(2)$. Hence,

$$
\left\|w_{v}\right\| \geqslant\|w\|-\left\|w_{h}\right\| \geqslant \lambda_{0}\left\|u_{v}\right\|-2\left\|w_{\pi}\right\| \geqslant\left(\lambda_{0}-2 \eta\right)\left\|u_{v}\right\| \quad \text { and } \quad\left\|w_{h}\right\| \leqslant 2 \eta\left\|u_{v}\right\| .
$$

We thus get $\left\|w_{v}\right\| \geqslant \frac{\lambda_{0}-2 \eta}{2 \eta}\left\|w_{h}\right\|>3 \beta\left\|w_{h}\right\|$ by our choice of the constant $\beta$.

4.1.3. Contraction of the tubular neighborhood. Let $d_{\pi}$ be the distance along the fibers of the tubular neighborhood $\pi: T \rightarrow \Sigma_{0}$, given by the induced metric. For any $m>0$ small, one denotes by $T_{m} \subset T$ the set of points $z \in T$ such that $d_{\pi}(z, \pi(z))<m$.

For any $z \in T_{m}$, one considers the geodesic segment joining $z$ to $\pi(z)$, parameterized by $[0,1]$. For each $\theta \in[0,1]$, this allows us to define $\theta \cdot z \in T_{m}$ as the barycenter of $z$ and $\pi(z)$ for the weights $\theta, 1-\theta$. For any $\theta \in[0,1]$ and $z \in T_{m}$, one defines $\theta \cdot z \in T_{m}$ as the barycenter of $z$ and $\pi(z)$ along the geodesic segment joining them in the fiber $\pi^{-1}(\pi(z))$. This gives a map $\Theta:(\theta, z) \mapsto \theta \cdot z$ on $[0,1] \times T_{m}$. For each $\theta \in[0,1]$, we also denote by $\Theta_{\theta}: T_{m} \rightarrow T_{m}$ the map $z \mapsto \theta \cdot z$.

Equivalently, let $\widetilde{F}$ be the tangent bundle on $T$ to the fibers of $\pi$, let $\mathcal{T}_{m}$ be the $m$-neighborhood of the zero section of the restriction $\widetilde{F}_{\mid} \Sigma_{0}$, and let $\widetilde{\Theta}:[0,1] \times \mathcal{T}_{m} \rightarrow \mathcal{T}_{m}$ be the map which sends $(\theta, v) \in[0,1] \times \widetilde{F}(x)$ on $\theta \cdot v$ in the vector space $\widetilde{F}(x)$. The maps $\Theta$ and $\widetilde{\Theta}$ are conjugated by the fibered exponential map.

Proposition 4.4. (1) The map $\Theta$ is $C^{1}$.

(2) The image of the derivative $D_{\theta} \Theta$ at a point $(\theta, z) \in[0,1] \times T_{m}$ is contained in the vertical space $V(z)$ of $z$. It has a norm bounded by the distance $d_{\pi}(z, \pi(z))$ in the fiber of $z$.

(3) For any $\rho>1$, if $m>0$ is small enough the following property holds.

For any $\theta \in[0,1]$, and any $z, z^{\prime} \in T_{m}$ such that $\pi(z)=\pi\left(z^{\prime}\right)$, we have $d_{\pi}(\theta \cdot z, \theta$. $\left.z^{\prime}\right) \leqslant \rho d_{\pi}\left(z, z^{\prime}\right)$.

(4) For any constants $\bar{\beta}>\beta^{\prime}>0$, if $m>0$ is small enough the following property holds. For any $\theta \in[0,1]$ and $z \in T_{m}$, the differential $D \Theta_{\theta}(z)$ sends $\mathcal{C}_{\beta^{\prime}}^{h}(z)$ inside $\mathcal{C}_{\bar{\beta}}^{h}(\theta \cdot z)$. 
Proof. The proposition may be obtained easily from the map $\widetilde{\Theta}$.

(1) The map $\widetilde{\Theta}$ is $C^{1}$, and so is $\Theta$.

(2) For any $v \in \mathcal{T}_{m}$, the map $\theta \mapsto \widetilde{\Theta}(\theta, v)$ is linear. The norm of its derivatives is equal to $\|v\|$. Let $z \in T_{m}$ be the image of $v$ by the fibered exponential map. Since the exponential map sends isometrically the line $\mathbb{R} \cdot v$ on the geodesic $\mathbb{R} . z$, one deduces that the derivative $D_{\theta} \Theta$ at the point $(\theta, z)$ has a norm equal to $\|v\|=d_{\pi}(z, \pi(z))$.

(3) For any $v, v^{\prime} \in \mathcal{T}_{m}$ which belong to the same fiber $\widetilde{F}(x)$ of $\widetilde{F}_{\mid \Sigma_{0}}$, and for any $\theta \in[0,1]$, we have $\left\|\theta \cdot v-\theta \cdot v^{\prime}\right\| \leqslant\left\|v-v^{\prime}\right\|$. We consider the images $z, z^{\prime} \in T_{m}$ of $v, v^{\prime}$ by the exponential map. At the point $(x, 0) \in \mathcal{T}_{m}$, the derivative of the exponential map is an isometry. Hence, for any $\rho>1$, if $v$ and $v^{\prime}$ are close enough to $(x, 0)$, we have $d_{\pi}\left(\theta \cdot z, \theta \cdot z^{\prime}\right) \leqslant \rho d_{\pi}\left(z, z^{\prime}\right)$.

(4) By construction, for any point $x \in \Sigma_{0}$, and any $\theta \in[0,1]$, the differential $D \Theta_{\theta}$ of the map $\Theta_{\theta}$ at $x$ maps the cone $\mathcal{C}_{\bar{\beta}}^{h}(x)$ into itself: the horizontal vectors at $x$ are preserved and the vertical vectors are contracted. The last item of the proposition thus follows by continuity of the differential of $\Theta$.

Remark 4.5. One can assume that the map $\pi$ and $\Theta$ are smooth on $\pi^{-1}\left(\Sigma_{0} \backslash K\right)$ and $[0,1] \times \pi^{-1}\left(\Sigma_{0} \backslash K\right)$. Indeed, $\Sigma_{0}$ can be taken smooth outside $K$ by Remark 3.3, and the vector field $\widetilde{F}$ tangent to the fibers of $\pi$ has been chosen smooth also.

4.1.4. Lipschitz functions and graphs over $\Sigma_{0}$. Let us consider $\lambda_{0}, \eta, \beta$ and a tubular neighborhood $\pi: T \rightarrow \Sigma_{0}$ as given by Proposition 4.2.

Definition 4.6. A function of $T$ is a map $h: U \rightarrow T$, where $U$ is a subset of $\Sigma_{0}$ such that $\pi \circ h(x)=x$ at any $x \in U$. The image $h(U)$ will be called the graph of $h$.

$h$ is $\beta$-Lipschitz if the tangent space $T_{z} h(U)$ (as in Definition 3.1) is contained in the cone $\mathcal{C}_{\beta}^{h}(z)$ for each $z \in U$.

The distance inside Lipschitz graphs is bounded.

Lemma 4.7. Let $\Sigma^{\prime} \subset \Sigma_{0}$ be a neighborhood of $K$ in $\Sigma_{0}$ and $h: \Sigma^{\prime} \rightarrow T$ a $\beta$-Lipschitz function. Then, for any curve $\sigma \subset \Sigma^{\prime}$, we have the estimate

$$
|h(\sigma)| \leqslant 2(1+\beta)|\sigma|
$$

, where $|\sigma|$ and $|h(\sigma)|$ are the lengths of the curves $\sigma$ and $h(\sigma)$.

Proof. At any point $z \in T$, any vector $u \in \mathcal{C}_{\beta}^{h}(z)$ decomposes as $u_{h}+u_{v}$ such that $\left\|u_{v}\right\| \leqslant$ $\beta\left\|u_{h}\right\|$. By Proposition $4.2(2)$, we also have $\left\|u_{h}\right\| \leqslant 2\|v\|$, where $v=D \pi(z) \cdot u$, so that $\|u\| \leqslant 2(1+\beta)\|v\|$. This gives the lemma.

The next lemma will show that the distance between Lipschitz graphs is contracted by $f^{-1}$.

Lemma 4.8. Fix a constant $\gamma>\left(\lambda_{0}-4 \eta(1+\beta)\right)^{-1}$ and a neighborhood $\Sigma^{\prime} \subset \Sigma_{0}$ of $K$ in $\Sigma_{0}$. Then, there exists a neighborhood $U_{\gamma} \subset T$ of $K$ which satisfies the following. 
For any graph $S$ of a $\beta$-Lipschitz function $h: \Sigma^{\prime} \rightarrow T$ and any points $z_{1}, z_{2}, \tilde{z}_{2}$ in $U_{\gamma}$ such that $z_{1}, \widetilde{z}_{2} \in S, \pi\left(z_{2}\right)=\pi\left(\widetilde{z}_{2}\right)$ and $\pi\left(f^{-1}\left(z_{1}\right)\right)=\pi\left(f^{-1}\left(z_{2}\right)\right)$, we have

$$
d_{\pi}\left(f^{-1}\left(z_{1}\right), f^{-1}\left(z_{2}\right)\right) \leqslant \gamma d_{\pi}\left(z_{2}, \tilde{z}_{2}\right) .
$$

Proof. One chooses $\lambda \in] 1, \lambda_{0}\left[\right.$ such that $\gamma>(\lambda-4 \eta(1+\beta))^{-1}$. Let $\sigma$ be a geodesic segment joining the points $f^{-1}\left(z_{1}\right)$ and $f^{-1}\left(z_{2}\right)$. Since $U_{\gamma}$ is small, the length of $\sigma$ is small.

The length $|f(\sigma)|$ is close to $\|D f(z) \cdot u\| \cdot|\sigma|$, where $z$ is a point of $\sigma$ and $u$ a unit vertical vector tangent to $\sigma$. Proposition 4.2(1) gives $|f(\sigma)| \geqslant \lambda_{0}|\sigma|$. The distance between $z_{1}$ and $z_{2}$ in $M$ is close to the length of $f(\sigma)$; hence

$$
d\left(z_{1}, z_{2}\right) \geqslant \lambda|\sigma|
$$

Similarly, the length of $\sigma^{\prime}=\pi \circ f(\sigma)$ is close to $\|D \pi \circ D f(z) \cdot u\| \cdot|\sigma|$, and Proposition 4.2(1) gives

$$
\left|\sigma^{\prime}\right| \leqslant 2 \eta|\sigma|
$$

Since $h$ is $\beta$-Lipschitz function, we have by Lemma 4.7 that

$$
\left|h\left(\sigma^{\prime}\right)\right| \leqslant 2(1+\beta)\left|\sigma^{\prime}\right| .
$$

Since the segment $h\left(\sigma^{\prime}\right)$ joins the points $z_{1}$ and $\widetilde{z}_{2}=h\left(\pi\left(z_{2}\right)\right)$, one gets by (7) and (6) the estimate $d\left(z_{1}, \tilde{z}_{2}\right) \leqslant 4 \eta(1+\beta)|\sigma|$. Writing $d\left(z_{1}, z_{2}\right) \leqslant d\left(z_{1}, \tilde{z}_{2}\right)+d\left(\tilde{z}_{2}, z_{2}\right)$, and using (5), one gets

$$
\lambda|\sigma| \leqslant 4 \eta(1+\beta)|\sigma|+d\left(\widetilde{z}_{2}, z_{2}\right) .
$$

Since $|\sigma|=d_{\pi}\left(f^{-1}\left(z_{1}\right), f^{-1}\left(z_{2}\right)\right)$, this gives as required

$$
d_{\pi}\left(f^{-1}\left(z_{1}\right), f^{-1}\left(z_{2}\right)\right) \leqslant(\lambda-4 \eta(1+\beta))^{-1} d\left(\tilde{z}_{2}, z_{2}\right) \leqslant \gamma d_{\pi}\left(z_{2}, \widetilde{z}_{2}\right) .
$$

4.1.5. Choice of the constants and of the tubular neighborhood. We explain the constants used in the proofs below and how to choose them.

(1) One chooses the constants required by Proposition 4.2: one first fixes $\left.\lambda_{0} \in\right] 1, \lambda_{K}[$, then $\eta>0$ small and finally $\beta, \delta \in] 0,1 / 2[$ small enough so that

$$
\beta<\frac{\lambda_{0}-2 \eta}{6 \eta}, \quad \lambda_{0}-4 \eta(1+\beta)>1 \text { and } \beta+\delta<\frac{1}{10} .
$$

(2) We also consider $\gamma>0$ and then $\rho>1$ such that

$$
\left(\lambda_{0}-4 \eta(1+\beta)\right)^{-1}<\gamma<1 \text { and } \gamma \rho<1 .
$$

The first part of (8) is used in Lemma 4.3. The second part guarantees the existence of $\gamma$ satisfying (8) as required in Lemma 4.8. The third part is used in the proof of Proposition 4.14 for the cone contraction. The constant $\rho>1$ appears in Proposition 4.4, and the condition $\gamma \rho<1$ will ensure the contraction of the graph transformation in the later constructions. 
(3) In order to obtain contraction of the horizontal cone fields, we choose $\bar{\beta} \in] \frac{\beta}{\lambda_{0}}$, $\beta$ [.

(4) Proposition 2.1 applied to $\Sigma$ gives $C_{\Sigma}>0$, and $c_{f}>1$ is any bound on $\|D f\|$ and $\left\|D f^{-1}\right\|$.

One can now fix the tubular neighborhood $T$ which satisfies the conclusions of Proposition 4.2 for the constants $\lambda_{0}, \eta, \beta$ and the conclusions of Proposition 4.4 for $\beta^{\prime}=\beta / \lambda_{0}$ and $\bar{\beta}$.

\subsection{A graph transformation}

4.2.1. Geometry of the graph images. Before defining the graph transformation, we need to check that the images by $f^{-1}$ of the Lipschitz graphs above $\Sigma_{0}$ remain Lipschitz graphs. We recall that $c_{f}>1$ is a constant which bounds $\|D f\|,\left\|D f^{-1}\right\|$.

Proposition 4.9. There exists a function $\varepsilon:] 0, m_{1}[\rightarrow] 0,+\infty[$, with the following properties. Let $\Sigma_{m}, \widehat{\Sigma}_{m}$ denote the open $\varepsilon(m)$ and $2 c_{f} \cdot \varepsilon(m)$-neighborhoods of $K$ in $\Sigma_{0}$. Then the following hold.

(1) $\lim _{m \rightarrow 0} \frac{m}{\varepsilon(m)}=0$.

(2) For any $m \in] 0, m_{1}\left[, f^{-1}\left(T_{m}\right) \cap \pi^{-1}\left(\widehat{\Sigma}_{m}\right) \subset T_{m}\right.$.

(3) For any $m \in] 0, m_{1}\left[\right.$, the image $f^{-1}(S)$ of the graph $S$ of any $\beta$-Lipschitz function $h: \widehat{\Sigma}_{m} \rightarrow T_{m}$ contains the graph of a $\frac{\beta}{\lambda_{0}}$-Lipschitz function $h^{\prime}: \Sigma_{m} \rightarrow T_{m}$ over $\Sigma_{m}$.

The proof uses two preliminary lemmas.

Lemma 4.10. For each small neighborhood $\Sigma^{\prime}$ of $K$ in $\Sigma_{0}$, there exists a positive constant $m=m\left(\Sigma^{\prime}\right)$ verifying the following property.

Consider the graph $S$ of a $\beta$-Lipschitz function $h: \Sigma^{\prime} \rightarrow T_{m\left(\Sigma^{\prime}\right)}$. Then, $f^{-1}(S)$ is contained in $T$ and is the graph of a function $h^{\prime}$ over the subset $\pi\left(f^{-1}(S)\right)$ of $\Sigma_{0}$.

Proof. Working with small charts (where the metric \|\| and the bundles $E$ and $F$ are almost constant) that cover the tubular neighborhood $T$, one gets the following property.

There exists $v>0$ such that, for any $\beta$-Lipschitz function $h: U \rightarrow T$ over a subset $U \subset \Sigma_{0}$, and for any points $x, y \in h(U)$ such that $d(x, y) \leqslant v$, then the geodesic segment joining $x$ to $y$ is tangent to the cone $\mathcal{C}_{2 \beta}^{h}$ at each point.

Since $f(K)=K$, since $\Sigma_{0}$ is tangent at each point $x \in K$ to $E(x)$, and since $E_{\mid K}$ is invariant by $D f$, there exists a neighborhood $\Sigma_{1} \subset \Sigma_{0}$ of $K$ in $\Sigma_{0}$ such that $f^{-1}\left(\Sigma_{1}\right)$ is contained in $T$ and is the graph of a function over a subset of $\Sigma_{0}$.

We now consider $\Sigma^{\prime} \subset \Sigma_{1}$ and prove that it satisfies the lemma with some constant $m>0$. Let us assume, by contradiction, that there exist a sequence $\left(m_{n}\right)$ going to 0 , a sequence of graphs $\left(S_{n}\right)$ of $\beta$-Lipschitz functions over $\Sigma^{\prime}$ such that $S_{n} \subset T_{m_{n}}$, and two sequences of points $\left(x_{n}\right)$ and $\left(y_{n}\right)$ such that, for each $n$, the points $x_{n}$ and $y_{n}$ are distinct, contained in $S_{n}$, and verify $\pi\left(f^{-1}\left(x_{n}\right)\right)=\pi\left(f^{-1}\left(y_{n}\right)\right)$.

We first prove that the distance $d\left(x_{n}, y_{n}\right)$ goes to 0 : in the other case, one would obtain, by considering some subsequences and using the fact that $m_{n}$ goes to 0 , two distinct points $x, y$ in $\Sigma^{\prime}$ whose images by $\pi \circ f^{-1}$ coincide, contradicting that $f^{-1}\left(\Sigma^{\prime}\right) \subset f^{-1}\left(\Sigma_{1}\right)$ is a graph. 
We consider the geodesic segment $\sigma_{n}$ joining $f^{-1}\left(x_{n}\right)$ to $f^{-1}\left(y_{n}\right)$ in the fiber of $\pi^{-1}$. Then, by Lemma 4.3 and our choice of $\beta$, the segment $f\left(\sigma_{n}\right)$, joining $x_{n}$ to $y_{n}$, is never tangent to the cones $\mathcal{C}_{3 \beta}^{h}$. On the other hand, for $n$ large enough, the points $x_{n}$ and $y_{n}$ are at distance less than $v$, so that the geodesic segment joining $x_{n}$ to $y_{n}$ is tangent to the cone $\mathcal{C}_{2 \beta}^{h}$. When $n$ tends to $\infty$, the angles between these two segments at $x_{n}$ go to 0 leading to a contradiction.

Lemma 4.11. There exists a function $\left.\varepsilon_{1}:\right] 0,+\infty[\rightarrow] 0,+\infty[$, with the following properties.

(1) $\lim _{m \rightarrow 0} \frac{m}{\varepsilon_{1}(m)}=0$.

(2) $T_{m}$ contains the image by $f^{-1}$ of the $\varepsilon_{1}(m)$-neighborhood of $K$ in $\Sigma_{0}$, for any $m>0$ small.

Proof. By Lemma 4.10, there is a neighborhood $\Sigma_{1}$ of $K$ in $\Sigma_{0}$ such that $f^{-1}\left(\Sigma_{1}\right)$ is the graph of a $C^{1}$-function $h$ which is tangent to the bundle $E$ at points of $K$. Hence, one can cover $K$ by finitely many open sets $U_{i}$ of $\Sigma_{0}$ and charts $\left.\varphi_{i}: U_{i} \rightarrow V_{i} \times\right] 0,1\left[{ }^{\operatorname{dim}(M)-d}\right.$ such that $V_{i}$ is an open set of $\Sigma_{0}$ and $h$ writes in this chart as a $C^{1}$-map from $V_{i}$ to $\left.V_{i} \times\right] 0,1\left[\operatorname{dim}(M)-d\right.$ of the form $x \mapsto\left(\pi(x), h_{i}(x)\right)$. Moreover, $h_{i}(x)$ and $D h_{i}(x)$ are equal to zero at points $x$ of $K$.

For any $\eta>0$, let $\nu_{0}(\eta)>0$ be the supremum of the norm of the derivatives of the maps $h_{i}$ on the $\eta$-neighborhood of $K$ in $\Sigma_{0}$. We set $v(\eta)=\max \left(\eta, v_{0}(\eta)\right)$. The map $\eta \mapsto v(\eta)$ is continuous, increasing, and goes to 0 with $\eta$ (since $D h_{i}$ vanishes at points of $K$ ). For $m$ small enough, one defines $\eta(m)>0$ as the minimum of the numbers $\eta$ such that $m \leqslant \eta v(\eta)$. Clearly, $\eta(m)$ goes to 0 with $m$ so that $\frac{m}{\eta(m)}=v(\eta(m))$ goes to 0 with $m$.

By construction (using the inequality $m \leqslant \eta v(\eta)$ and the facts that $h$ vanishes on $K$ and has a derivative bounded by $v(\eta)$ on the $\eta$-neighborhood of $K$ ), the $C^{0}$-norm of $h$ is bounded by $m$ on the $\eta(m)$-neighborhood of $K$ in $\Sigma_{0}$. In other words, the graph $f^{-1}(S)$ over the $\eta(m)$-neighborhood of $K$ in $\Sigma_{0}$ is contained in $T_{m}$. The differential of $f^{-1}$ is bounded by $c_{f}>1$. Hence, the function $\varepsilon_{1}(m)=\frac{\eta(m)}{c_{f}}$ satisfies the statement of the lemma.

Proof of Proposition 4.9. We set $\varepsilon(m)=\frac{1}{2 c_{f}} \varepsilon_{1}(m)$ with $\varepsilon_{1}$ as in Lemma 4.11.

(1) The first item of the lemma is an easy consequence of Lemma 4.11(1).

(2) Let $z_{1}^{\prime}$ be a point in $f^{-1}\left(T_{m}\right) \cap \pi^{-1}\left(\widehat{\Sigma}_{m}\right)$ and $z_{2}^{\prime} \in f^{-1}\left(\Sigma_{1}\right)$ such that $x_{1}^{\prime}:=\pi\left(z_{1}^{\prime}\right)=$ $\pi\left(z_{2}^{\prime}\right)$. We also let $z_{1}:=f\left(z_{1}^{\prime}\right) \in T_{m}$ and $\tilde{z}_{1} \in \Sigma_{0}$ such that $\pi\left(\tilde{z}_{1}\right)=\pi\left(z_{1}\right)$. Since $z_{1} \in T_{m}$, we have $d_{\pi}\left(z_{1}, \widetilde{z}_{1}\right) \leqslant m$. By Lemma 4.8 , we have

$$
d_{\pi}\left(z_{1}^{\prime}, z_{2}^{\prime}\right) \leqslant \gamma d_{\pi}\left(z_{1}, \tilde{z}_{1}\right) \leqslant \gamma m .
$$

Since $\Sigma$ and $f^{-1}(\Sigma)$ are tangent at points of $K$, for $m$ small we have

$$
d_{\pi}\left(x_{1}^{\prime}, z_{2}^{\prime}\right) \leqslant d\left(z_{1}^{\prime}, K\right) \leqslant 2 c_{f} \varepsilon(m) \leqslant(1-\gamma) \cdot m
$$

by Lemma $4.11(1)$. This proves $d_{\pi}\left(x_{1}^{\prime}, z_{1}^{\prime}\right) \leqslant m$ and gives the second item.

(3) We now prove the following property:

$$
f^{-1}\left(T_{m} \cap \pi^{-1}\left(\partial \widehat{\Sigma}_{m}\right)\right) \cap \pi^{-1}\left(\Sigma_{m}\right)=\emptyset .
$$


We consider a point $z_{1}$ in $T_{m} \cap \pi^{-1}\left(\partial \widehat{\Sigma}_{m}\right)$ and $z_{2}=\pi\left(z_{1}\right)$. Let $z_{1}^{\prime}, z_{2}^{\prime}$ be their images by $f^{-1}$, and let $x_{1}^{\prime}, x_{2}^{\prime}$ be the projections of $z_{1}^{\prime}, z_{2}^{\prime}$ by $\pi$. It is enough to show that $x_{1}^{\prime}$ and $K$ are at distance larger than $\varepsilon(m)$ in $\Sigma_{0}$. In particular, one can assume that $x_{1}^{\prime} \in \widehat{\Sigma}_{m}$.

The distance between $z_{2}$ and $K$ in $\Sigma_{0}$ equals $2 c_{f} \varepsilon(m)$; hence the distance between $z_{2}^{\prime}$ and $K$ is larger than $2 \varepsilon(m)$ in $f^{-1}\left(\Sigma_{0}\right)$ and (since $f^{-1}(\Sigma)$ and $\Sigma$ are tangent at points of $K$ ) the distance between $x_{2}^{\prime}$ and $K$ is larger than $\frac{3}{2} \varepsilon(m)$. We will show that the distance between $x_{1}^{\prime}$ and $x_{2}^{\prime}$ in $\Sigma_{0}$ is smaller than $\frac{\varepsilon(m)}{2}$, and this will give the announced property.

Since $x_{1}^{\prime} \in \widehat{\Sigma}_{m}$, by the second item one has $z_{1}^{\prime} \in T_{m}$, and hence $d_{\pi}\left(x_{1}^{\prime}, z_{1}^{\prime}\right) \leqslant m$. Since $d_{\pi}\left(z_{1}, z_{2}\right) \leqslant m$, we have $d\left(z_{1}^{\prime}, z_{2}^{\prime}\right) \leqslant 2 c_{f} m$, where $c_{f}$ bounds the derivative of $f^{-1}$. Since $z_{2} \in \widehat{\Sigma}_{m}$, it belongs to the $2 c_{f} \varepsilon(m)$-neighborhood of $K$ in $\Sigma_{0}$. We have $2 c_{f} \varepsilon(m) \leqslant \varepsilon_{1}(m)$, and hence $z_{2}^{\prime} \subset T_{m}$ by Lemma $4.11(2)$; that is, $d_{\pi}\left(z_{2}^{\prime}, x_{2}^{\prime}\right)$ is less than $m$. The distance between $x_{1}^{\prime}$ and $x_{2}^{\prime}$ is thus bounded by $2\left(1+c_{f}\right) m$.

Since $\frac{m}{\varepsilon(m)}$ may be taken arbitrarily small, one deduces that the distance in $\Sigma_{0}$ between $x_{1}^{\prime}$ and $x_{2}^{\prime}$ is smaller than $\frac{\varepsilon(m)}{2}$, as required, proving (11).

Let us come to the proof of the last item. By Lemma 4.10, the image by $f^{-1}$ of the graph $S$ of the function $h$ over $\widehat{\Sigma}_{m}$ is the graph $S^{\prime}$ of a function $h^{\prime}$ defined over a subset $D$ of $\Sigma_{0}$. By Proposition 4.2(3), $h^{\prime}$ is $\frac{\beta}{\lambda_{0}}$-Lipschitz. By the second item, $S^{\prime} \cap \pi^{-1}\left(\Sigma_{m}\right)$ is contained in $T_{m}$. By (11), $\Sigma_{m}$ and the boundary of $D$ do not intersect. Hence, $\Sigma_{m}$ is contained in $D$. This shows that $S^{\prime}$ contains the graph of a function defined over $\Sigma_{m}$.

4.2.2. Definition of the graph transformation. For $m$ small, we denote the following.

$U_{m}$ : the open set $T_{m} \cap(\pi \circ f)^{-1}\left(\Sigma_{m}\right)$. For $m$ small it is an arbitrarily small neighborhood of $K$.

Lip $_{m, \beta}$ : the set of $\beta$-Lipschitz functions $h: \Sigma_{0} \rightarrow T_{m}$, which vanish outside $\Sigma_{m}$, i.e., $h(x)=x$ for $x \in \Sigma_{0} \backslash \Sigma_{m}$. It is endowed with the $C^{0}$-distance: if $h, h^{\prime} \in \operatorname{Lip}_{m, \beta}$ are two Lipschitz functions, we set

$$
d\left(h, h^{\prime}\right)=\sup _{x \in \Sigma_{m}} d_{\pi}\left(h(x), h^{\prime}(x)\right) .
$$

By Arzela-Ascoli's theorem, this space is compact.

$\operatorname{Lip}_{m, \beta}(K)$ : the subset of functions $h \in \operatorname{Lip}_{m, \beta}$ vanishing on $K$ (i.e. $\forall x \in K, h(x)=x$ ).

$\varphi_{m}$ : a smooth function $\Sigma_{0} \rightarrow[0,1]$, given by Proposition 2.1 , equal to 1 in the $\frac{\varepsilon(m)}{2}$-neighborhood of $K$ and to 0 in a neighborhood of $\Sigma_{0} \backslash \Sigma_{m}$. Its derivative is bounded by $\frac{2 C_{\Sigma}}{\varepsilon(m)}$, where $C_{\Sigma}$ is the constant associated to the manifold $\Sigma$.

$V_{m}$ : an open neighborhood of $\Sigma_{0} \backslash \Sigma_{m}$ in $\Sigma_{0}$ where $\varphi_{m}$ vanishes.

$\phi_{m}(h)$ : the function $\Sigma_{0} \rightarrow T_{m}$ associated to a function $h: \Sigma_{m} \rightarrow T_{m}$ as follows: it is equal to $\varphi_{m} \cdot h$ on $\Sigma_{m}$ and to the identity outside $\Sigma_{m}$. With the notation of $\S 4.1 .3$, $\phi_{m}(h)(x)=\Theta\left(\varphi_{m}(x), h(x)\right)$ for each point $x \in \Sigma_{m}$. 
$G_{m}(h)$ : the function $\Sigma_{0} \rightarrow T_{m}$ associated to $h \in \operatorname{Lip}_{m, \beta}$ as follows: by Proposition 4.9(3), the image of the graph of $h$ by $f^{-1}$ contains the graph of a Lipschitz function $h^{\prime}: \Sigma_{m} \rightarrow T_{m}$. We set $G_{m}(h)=\phi_{m}\left(h^{\prime}\right)$.

The map $G_{m}$ is called the graph transformation.

One can see the graph $S^{\prime}$ of $G_{m}(h)$ as the image of the graph $S$ of $h$ by some $C^{1}$-map. Indeed, if one defines in $f(T)$ the map

$$
\Psi_{m}: z \mapsto \Theta\left(\varphi_{m} \circ \pi \circ f^{-1}(z), f^{-1}(z)\right),
$$

then the graph $S^{\prime}$ is the union of $\Psi_{m}(S) \cap \pi^{-1}\left(\Sigma_{m}\right)$ with $\Sigma_{0} \backslash \Sigma_{m}$.

By construction, $S^{\prime}$ and $\Sigma_{0}$ coincide on the open set $V_{m} \subset \Sigma_{0}$. Note that $S^{\prime}$ is the union of $V_{m}$ with $\Psi_{m}\left(S \cap U_{m}\right)$.

Remark 4.12. From Remarks 3.3 and 4.5, one can construct the map $\Psi_{m}$ as smooth as the diffeomorphism $f$.

4.2.3. Invariance of the space of Lipschitz graphs. The next proposition shows that the image $G_{m}(h)$ of a Lipschitz graph is also Lipschitz.

Proposition 4.13. For $m>0$ small, $G_{m}$ preserves Lip $_{m, \beta}$.

This follows immediately from the next result.

Proposition 4.14. For $m>0$ small, and $z \in T_{m} \cap f(T)$, the image of the cone $\mathcal{C}_{\beta}^{h}(z)$ is contained in the cone $\mathcal{C}_{\beta}^{h}\left(\Psi_{m}(z)\right)$. Moreover, $D \Psi_{m}(z) \cdot u_{0}$ does not vanish for $u_{0} \in \mathcal{C}_{\beta}^{h}(z)$.

Proof. Let us fix $u_{0} \in \mathcal{C}_{\beta}^{h}(z)$ and denote $u_{1}=D \Psi(z) \cdot u_{0}$. We also set $\tilde{z}_{1}=f^{-1}(z)$ and $\tilde{u}_{1}=D f^{-1}(z) \cdot u_{0}$. By Proposition $4.2(3), \widetilde{u}_{1}$ belongs to $\mathcal{C}_{\frac{\beta}{\lambda_{0}}}^{h}\left(\widetilde{z}_{1}\right)$. Note that $u_{1}$ is the image of $\tilde{u}_{1}$ by the tangent map at $\tilde{z}_{1}$ of

$$
P_{m}: x \mapsto \Theta\left(\varphi_{m} \pi(x), x\right) .
$$

We aim to compare $u_{1}$ with $\tilde{u}_{1}$.

Claim 4.15. If $m$ is small, for any $\widetilde{z}_{1} \in f^{-1}\left(T_{m}\right)$ and $\tilde{u}_{1} \in \mathcal{C}_{\frac{\beta}{\lambda_{0}}}^{h}$, we have

$$
\left|\left\|D P_{m} \cdot \tilde{u}_{1}\right\|-\left\|\tilde{u}_{1}\right\|\right| \leqslant 9(\beta+\delta) \cdot\left\|\tilde{u}_{1}\right\| .
$$

Proof. If $\widetilde{z}_{1}$ belongs to $\pi^{-1}\left(\Sigma \backslash \Sigma_{m}\right)$, then $P_{m}$ coincides locally with the projection $\pi$ on $\Sigma_{0}$. In particular, the image of $D P_{m}\left(\widetilde{z}_{1}\right)$ coincides with the tangent space to $\Sigma_{0}$, and hence is contained in $\mathcal{C}_{\beta}^{h}\left(\Psi_{m}(z)\right)$, and by Proposition $4.2(2)$ we have

$$
\left|\left\|D P_{m} \cdot \tilde{u}_{1}\right\|-\left\|\tilde{u}_{1}\right\|\right| \leqslant \delta\left\|\tilde{u}_{1}\right\| .
$$

Otherwise, $\tilde{z}_{1}$ belongs to $f^{-1}\left(T_{m}\right) \cap \pi^{-1}\left(\Sigma_{m}\right)$, and it also belongs to $T_{m}$ by Proposition $4.9(2)$, so that denoting $x_{1}=\pi\left(\widetilde{z}_{1}\right)$ we have

$$
d_{\pi}\left(\widetilde{z}_{1}, x_{1}\right) \leqslant m
$$


We set $\tilde{u}_{1, \pi}=D \pi\left(\widetilde{z}_{1}\right) \cdot \tilde{u}_{1}$ and $\theta_{1}=\varphi_{m}\left(x_{1}\right)$. The image $u_{1}:=D P_{m}(z) \cdot \tilde{u}_{1}$ is equal to $D \Theta\left(\theta_{1}, \widetilde{z}_{1}\right) .\left(D \varphi_{m} \cdot \tilde{u}_{1, \pi}, \tilde{u}_{1}\right)$, and it decomposes as

$$
u_{1}=v+w=D_{\theta} \Theta\left(\theta_{1}, \widetilde{z}_{1}\right) \circ D \varphi_{m}\left(x_{1}\right) \cdot \tilde{u}_{1, \pi}+D \Theta_{\theta_{1}}\left(\widetilde{z}_{1}\right) \cdot \tilde{u}_{1},
$$

where $\Theta_{\theta_{1}}$ is the map $z \mapsto \Theta\left(\theta_{1}, z\right)$ as before.

By Proposition 4.4(2), the vertical vector $v$ has a norm bounded by $d_{\pi}\left(\widetilde{z}_{1}, x_{1}\right)\left\|D \varphi_{m}\right\|\left\|\widetilde{u}_{1, \pi}\right\|$. The first term of this product is bounded by $m$ by (14) and the second by $\frac{2 C_{\Sigma}}{\varepsilon(m)}$. Hence,

$$
\|v\| \leqslant \frac{2 C_{\Sigma} m}{\varepsilon(m)}\left\|\tilde{u}_{1, \pi}\right\|
$$

The second vector $w=D \Theta_{\theta_{1}}\left(\widetilde{z}_{1}\right) \cdot \widetilde{u}_{1}$ decomposes as the sum $w_{h}+w_{v}$ of a horizontal vector and a vertical vector. By construction, $\pi \circ \Theta_{\varphi\left(x_{1}\right)}=\pi$, so that $D \pi\left(z_{1}\right) \cdot w_{h}=$ $D \pi\left(\widetilde{z}_{1}\right) \cdot \widetilde{u}_{1}=\tilde{u}_{1, \pi}$. By Proposition $4.2(2),\left\|w_{h}\right\| \geqslant(-\delta)\left\|\tilde{u}_{1, \pi}\right\|$. One the other hand, using that $\tilde{u}_{1}$ is contained in a cone $\mathcal{C}_{\beta / \lambda_{0}}^{h}$, and $\bar{\beta}>\beta / \lambda_{0}$, Proposition 4.4(4) implies that $w$ belongs to a cone $\mathcal{C}_{\bar{\beta}}^{h}$. Hence, $\left\|w_{v}\right\| \leqslant \bar{\beta}\left\|w_{h}\right\|$.

Let us recall that $\bar{\beta}<\beta$. By these estimates and (15), the vertical component $u_{1, v}=$ $v+w_{v}$ of $u_{1}$ is bounded by

$$
\left\|u_{1, v}\right\| \leqslant\|v\|+\left\|w_{v}\right\| \leqslant\left(\frac{4 C_{\Sigma^{m}}}{\varepsilon(m)}+\bar{\beta}\right)\left\|w_{h}\right\| .
$$

The horizontal component $u_{1, h}$ of $u_{1}$ is $w_{h}$.

If one chooses $m$ small enough, $\frac{2 C_{\Sigma}}{\varepsilon(m)} m$ may be assumed arbitrarily small by Proposition $4.9(1)$, so that $\frac{2 C_{\Sigma}}{\varepsilon(m)} m+\bar{\beta}$ is less than $\beta$. Hence $u_{1}$ belongs to the cone $\mathcal{C}_{\beta}^{h}\left(z_{1}\right)$. Moreover, we have

$$
\left|\left\|u_{1}\right\|-\left\|\widetilde{u}_{1}\right\|\right| \leqslant\left|\left\|\tilde{u}_{1}\right\|-\left\|D \Theta_{\theta_{1}}\left(\widetilde{z}_{1}\right) \cdot \tilde{u}_{1}\right\|\right|+\frac{2 C_{\Sigma} m}{\varepsilon(m)}\left\|\tilde{u}_{1, \pi}\right\| .
$$

For $m$ small, $z_{1}:=P_{m}\left(\widetilde{z}_{1}\right)$ and $\widetilde{z}_{1}$ are arbitrarily close. Furthermore, $\widetilde{u}_{1}$ and $D \Theta_{\theta_{1}}\left(\widetilde{z}_{1}\right) \cdot \widetilde{u}_{1}$ have the same projection by $D \pi$ and are tangent to $\mathcal{C}_{\beta}^{h}$. Recalling that $\left.\beta, \delta \in\right] 0,1 / 2[$, and using Proposition 4.2(2), we also deduce that

$$
\left|\left\|\tilde{u}_{1}\right\|-\left\|D \Theta_{\theta_{1}}\left(\tilde{z}_{1}\right) \cdot \tilde{u}_{1}\right\|\right| \leqslant 8(\beta+\delta)\left\|\tilde{u}_{1}\right\| .
$$

The claim is thus proved in all the cases.

We have proved that $u_{1}$ belongs to $\mathcal{C}_{\beta}^{h}$, which gives the first part of the Proposition 4.14 (and the first item of the Definition 2.4). Note that, if $u_{0}$ is non-zero, the same holds for $\tilde{u}_{1}=D f^{-1}(z) \cdot u_{0}$. If $\beta+\delta<1 / 10$, estimate (13) gives $\left\|u_{1}\right\| \geqslant \frac{1}{10}\left\|\tilde{u}_{1}\right\|$; hence $u_{1}$ does not vanish. In particular, we have obtained the second part of the proposition (and the second item of the Definition 2.4).

In order to control the smoothness of the center manifold we will need the following additional result, which can be skipped at a first reading.

Addendum 4.16. Let us assume that $K$ is $r$-normally hyperbolic. One can choose the tubular neighborhood $\pi: T \rightarrow \Sigma_{0}$ such that, for $\beta>0$ and $m>0$ small enough, the cone $\mathcal{C}_{\beta}^{h}$ is r-contracted by the restriction of $\Psi_{m}$ to $U_{m}$. 
Proof. Note that the two first items of the Definition 2.4 are satisfied. In order to get the third one, one has to choose the tubular neighborhood $\pi: T \rightarrow \Sigma_{0}$ carefully.

Since $K$ is $r$-normally hyperbolic and the decomposition $T M_{\mid K}=E^{c} \oplus E^{u u}$ is dominated, there exists $n_{K} \geqslant 1$ such that, for $x \in K$ and each unit vector $u^{c} \in E^{c}(x)$, $v^{u} \in E^{u u}(x)$,we have

$$
\min \left(\left\|D f^{-n_{K}}(x) \cdot u^{c}\right\|,\left\|D f^{-n_{K}}(x) \cdot u^{c}\right\|^{r}\right)>3\left\|D f^{-n_{K}}(x) \cdot v^{u}\right\| .
$$

The same holds for any unit vectors in some thin continuous cone fields $\mathcal{C}^{c} \mathcal{C}^{u u}$ containing the bundles $E^{c}, E^{u u}$, respectively, and defined on a neighborhood of $K$.

One will choose the cone field $\mathcal{C}^{u u}$ to be invariant by $D f$. For instance, for $a>0$ small enough, one may consider at points $x \in K$ the cone $\mathcal{C}^{u u}(x):=\mathcal{C}_{a}^{u u}(x)$ of vectors $w \in T_{x} M$ such that the norm of the component along $E^{c}(x)$ is smaller than $a$ times the norm of the component along $E^{u u}(x)$ : then the image of $\mathcal{C}_{a}^{u u}(x)$ by $D f^{-1}$ is contained in $\widetilde{\mathcal{C}^{u u}}(f(x)):=\mathcal{C}_{a / \lambda_{0}}^{u u}(f(x))$. The cone fields $\mathcal{C}^{u u}$ and $\widetilde{\mathcal{C}^{u u}}$ may be extended continuously to a neighborhood of $K$.

Let us choose $b>0$ small. The cones $\mathcal{C}^{u u}$ and $\widetilde{\mathcal{C}^{u u}}$ being chosen as above with respect to the $r$-normal hyperbolicity and the domination, we assume that the tangent spaces to the fibers of $F$ are close enough to the bundle $E^{u u}$. Moreover, $D P_{m}$ contracts the fibers of $\pi$, while its restriction to $E_{\mid K}^{c}$ is the identity. Consequently, for any points $x \in K$ and any $m$ small, if we have $D P_{m}(x) \cdot u \in \widetilde{\mathcal{C}^{u} u}(x)$ then $D P_{m}(x) \cdot u$ and $u$ are almost collinear, $u$ belongs to $\mathcal{C}^{u u}(x)$, and $\left\|D P_{m}(x) \cdot u\right\|$ is smaller than $(1+b) \cdot\|u\|$. In particular, for any $z \in U_{m}$ and $u \in T_{z} M$, we have

$$
D \Psi_{m}(z) \cdot u \in \mathcal{C}^{u u}(z) \Rightarrow\left\{\begin{array}{l}
u \in \mathcal{C}^{u u}(z), \\
\left\|D \Psi_{m}(z) \cdot u\right\| \leqslant(1+b)\left\|D f^{-1}(z) \cdot u\right\| \\
\left\|\frac{D \Psi_{m}(z) \cdot u}{\left\|D \Psi_{m}(z) \cdot u\right\|}-\frac{D f^{-1}(z) \cdot u}{\left\|D f^{-1}(z) \cdot u\right\|}\right\| \leqslant b .
\end{array}\right.
$$

Arguing in a similar way, the cone field $\widetilde{\mathcal{C}^{u u}}$ has the same properties.

If $\beta, m>0$ are small and $y$ is close to $K$, any unit vector $u \in \mathcal{C}_{\beta / \lambda_{0}}^{h}(y)$ is close to its image by $D P_{m}(y)$ by Claim 4.15 ; moreover, the cones $\mathcal{C}_{\beta}^{h}$ are contained in the cones $\mathcal{C}^{c}$. In particular, we have, for any $z \in U_{m}$,

$$
u \in \mathcal{C}_{\beta}^{h}(z) \Rightarrow\left\{\begin{array}{l}
D \Psi_{m}(z) \cdot u \in \mathcal{C}_{\beta}^{h}\left(\Psi_{m}(z)\right) \\
\left\|D \Psi_{m}(z) \cdot u\right\| \geqslant(1-b)\left\|D f^{-1}(z) \cdot u\right\| \\
\left\|\frac{D \Psi_{m}(z) \cdot u}{\left\|D \Psi_{m}(z) \cdot u\right\|}-\frac{D f^{-1}(z) \cdot u}{\left\|D f^{-1}(z) \cdot u\right\|}\right\| \leqslant b
\end{array}\right.
$$

In particular, for any $z \in U_{m} \cap \cdots \cap \Psi_{m}^{-n_{K}+1}(U)$ and any unit vectors $u, v$ with $u \in \mathcal{C}_{\beta}^{h}(z)$ and $D \Psi_{m}^{n_{K}}(z) \cdot v \in \mathcal{C}^{u u}\left(\Psi_{m}^{n_{K}}(z)\right)$, we have

$$
\min \left(\left\|D \Psi_{m}^{n_{K}}(z) \cdot u\right\|,\left\|D \Psi_{m}^{n_{K}}(z) \cdot u\right\|^{r}\right)>2\left\|D \Psi_{m}^{n_{K}}(z) \cdot v\right\| .
$$


Let us now consider $n \geqslant 1$ large, $z \in U_{m} \cap \cdots \cap \Psi_{m}^{-n+1}(U)$, and any unit vectors $u, v$ with $u \in \mathcal{C}_{\beta}^{h}(z)$ and $D \Psi_{m}^{n}(z) \cdot v \notin \mathcal{C}_{\beta}^{h}\left(\Psi_{m}^{n}(z)\right)$.

One can decompose $v$ as a sum $v^{c}+v^{u}$ such that $D \Psi_{m}^{n}(z) \cdot v^{u} \in \widetilde{\mathcal{C}^{u u}}$ and $D \Psi_{m}^{n}(z) \cdot v^{c} \in$ $D \Psi_{m}^{n}\left(\mathcal{C}_{\beta / \lambda_{0}}^{h}\right)$. Note that, since the image of $v$ is not in $\mathcal{C}_{\beta}$, there exists $\widetilde{C}>0$ uniform such that

$$
\left\|D \Psi_{m}^{n}(z) \cdot v^{u}\right\| \geqslant \widetilde{C}^{-1}\left\|D \Psi_{m}^{n}(z) \cdot v^{c}\right\| .
$$

If $n_{0}=\ell n_{K}$, we have by (16) that

$$
\left\|D \Psi_{m}^{n-n_{0}}(z) \cdot v^{c}\right\| \leqslant \widetilde{C} 2^{-\ell}\left\|D \Psi_{m}^{n-n_{0}}(z) \cdot v^{u}\right\| .
$$

Since $D \Psi_{m}^{n-n_{0}}(z) \cdot v^{u}$ belongs to $\widetilde{\mathcal{C}^{u u}}$, one deduces that $D \Psi_{m}^{n-n_{0}}(z) \cdot v$ belongs to $\mathcal{C}^{u u}$. One can thus apply 16 to any iterate $D \Psi_{m}^{k} \cdot u, D \Psi_{m}^{k} \cdot v$ such that $0 \leqslant k \leqslant n-n_{0}-n_{K}$, and prove for some $C>0$ uniform the required estimate

$$
\left\|D \Psi_{m}^{n}(x) \cdot v\right\| \leqslant C 2^{-n / n_{K}} \min \left(\left\|D \Psi_{m}^{n}(x) \cdot u\right\|,\left\|D \Psi_{m}^{n}(x) \cdot u\right\|^{r}\right) .
$$

\subsection{Fixed point of the graph transformation}

\subsubsection{Existence of the fixed point.}

Proposition 4.17. For $m>0$ small enough, $G_{m}$ has a unique fixed point in Lip $p_{m, \beta}$.

Since $\operatorname{Lip}_{m, \beta}$ is compact, the next lemma implies Proposition 4.17.

Lemma 4.18. For $m$ small, $G_{m}$ is a contraction of $\mathrm{Lip}_{m, \beta}$.

Proof. Let us consider two Lipschitz functions $h_{1}, h_{2} \in \operatorname{Lip}_{m, \beta}$. By Proposition 4.9(3), the images by $f^{-1}$ of their graphs contain the graphs of two Lipschitz functions $h_{1}^{\prime}, h_{2}^{\prime}$ : $\Sigma_{m} \rightarrow T_{m}$. Let $x$ be a point in $\Sigma_{m}$. One first wants to estimate the length $d_{\pi}\left(h_{1}^{\prime}(x), h_{2}^{\prime}(x)\right)$.

Let us denote by $z_{1}$ and $z_{2}$ the images by $f$ of $h_{1}^{\prime}(x)$ and $h_{2}^{\prime}(x)$. We introduce their projections $x_{1}, x_{2} \in \Sigma_{m}$ by $\pi$, so that $z_{1}=h_{1}\left(x_{1}\right)$ and $z_{2}=h_{2}\left(x_{2}\right)$. We also consider the point $\widetilde{z}_{2}=h_{1}\left(x_{2}\right)$. By Lemma 4.8 , we have

$$
d_{\pi}\left(h_{1}^{\prime}(x), h_{2}^{\prime}(x)\right) \leqslant \gamma d\left(h_{1}, h_{2}\right) .
$$

By Proposition 4.4(3), we also have

$$
d_{\pi}\left(\varphi_{m}(x) \cdot h_{1}^{\prime}(x), \varphi_{m}(x) \cdot h_{2}^{\prime}(x)\right) \leqslant \rho d_{\pi}\left(h_{1}^{\prime}(x), h_{2}^{\prime}(x)\right) .
$$

Thus, one gets

$$
d\left(G_{m}\left(h_{1}\right), G_{m}\left(h_{2}\right)\right) \leqslant \gamma \rho d\left(h_{1}, h_{2}\right) .
$$

We have chosen $\gamma \rho<1$, so this implies the contraction property for $G_{m}$.

4.3.2. $C^{1}$-smoothness of the fixed graph. In order to prove that the graph $S$ of the function $h \in \operatorname{Lip}_{m, \beta}$ fixed by $G_{m}$ is $C^{1}$, we apply the following proposition to the tubular neighborhood $\pi: T_{m} \rightarrow \Sigma_{0}$, the graph $S$, the map $\Psi_{m}$, and the open sets $U_{m}, V_{m}$.

Proposition 4.19. Let us consider a $C^{1}$ submersion $\pi: T \rightarrow \Sigma_{0}$ and a section $S$. We assume furthermore that 
(1) there exists a $C^{1}-$ map $\Psi: U \rightarrow T$ defined on an open set $U \subset T$, which preserves $S$ : the restriction of $\Psi$ to $S \cap U$ is a homeomorphism to its image and $\Psi(S \cap U) \subset S$,

(2) $S$ is $C^{1}$ on an open set $V$ of $S$ containing $S \backslash \Psi(S \cap U)$, and

(3) there exists a continuous cone field $\mathcal{C}$ on $T$ of dimension $d=\operatorname{dim}\left(\Sigma_{0}\right)$ that is transverse to the fibration $\pi$, contracted by $\Psi$, and such that $S$ is tangent to $\mathcal{C}$.

Then $S$ is a $C^{1}$-submanifold.

Corollary 4.20. The fixed point $h: \Sigma_{0} \rightarrow T$ of the map $G_{m}$ is a $C^{1}$ function.

Proof of Proposition 4.19. Let us consider a point $z_{0} \in S$. There are two cases.

- Case 1. There exist an integer $n \geqslant 0$ and a point $z_{-n} \in S \cap V$ such that, for each $0 \leqslant k<n$, the point $\Psi^{k}\left(z_{-n}\right)$ belongs to $S \cap U$ and $\Psi^{n}\left(z_{-n}\right)=z_{0}$. Since $z_{-n}$ belongs to $V$, the graph $S$ is $C^{1}$ in a neighborhood of $z_{-n}$. By the definition of contracted cone fields, the restriction of $D \Psi^{n}$ to the tangent bundle of $S \cap V$ is non-degenerate: the restriction of $\Psi$ to a neighborhood of $z_{0}$ in $S$ is a diffeomorphism to its image, and by invariance $S$ is $C^{1}$ in a neighborhood of $z_{0}$. In particular, $S$ is tangent to a $d$-dimensional space $L_{z} \subset T_{z} M$ transverse to $\pi$ at each point $z$ close to $z_{0}$.

- Case 2. There exists an infinite sequence $\left(z_{-n}\right)_{n \in \mathbb{N}}$ of points in $S \cap U$ such that $\Psi\left(z_{-n}\right)=z_{-n+1}$ for each $n \geqslant 1$. In this case, using that $\Psi_{\mid S \cap U}$ is a homeomorphism on its image, for each $n \geqslant 0$, the graph $S$ is tangent to the continuous cone field $\mathcal{C}^{n}=D \Psi^{n} \cdot \mathcal{C}$ in a neighborhood of $z_{0}$. This cone field is exponentially thin around a $d$-dimensional subspace of $T_{z_{0}} M$ by Lemma 2.6. The intersection of the $\mathcal{C}^{n}\left(z_{0}\right)$ is thus a $d$-dimensional space $L_{z_{0}}$ and $S$ is tangent to $L_{z_{0}}$ at $z_{0}$.

We now prove that $z \mapsto L_{z}$ is continuous at any point. It is clear in the first case. In the second case, it comes from the fact that $L_{z}$ is tangent to the thin continuous cone field $\mathcal{C}^{n}$ in a neighborhood of $z_{0}$, for arbitrarily large $n$.

We have thus proved that the section $S$ of $\pi$ has continuous tangent spaces transverse to $\pi$ : this is a $C^{1}$ transverse section, and hence a $C^{1}$-submanifold.

Under stronger assumptions, we can prove a higher smoothness. It is not used in the proof of Theorem 4.1, and may be skipped at a first reading.

Addendum 4.21. Under the assumptions of the Proposition 4.19, let us suppose furthermore that, for some $\alpha \in(0,1]$, the map $\Psi$ and the manifolds $S \cap V$ are $C^{1, \alpha \text {, }}$ and that the cone field $\mathcal{C}$ is $(1+\alpha)$-contracted. Then $S$ is the graph of a $C^{1, \alpha}$ function.

Proof. Let us consider a point $z \in S$ and a point $z^{\prime} \in S$ close to $z$. We have to estimate the difference between the slopes of $T_{z} S$ and $T_{z^{\prime}} S$.

In the following, we define for $x \in S$ the distance $d\left(u, T_{x} S\right)$ between $T_{x} S$ and a non-zero vector $u \in T_{x} M$ as the norm of the linear projection of the unit vector $u /\|u\|$ to the tangent space of the fiber of $\pi$ containing $x$, parallel to $T_{x} S$.

By Claim 2.7, the angle between $T_{x} S$ and the fiber containing $x$ is uniformly bounded away from zero; hence the linear projection on the tangent space of the fiber at $x$ and parallel to $T_{x} S$ has a norm bounded by a constant $C_{1}>0$. Similarly, for any point $x \in$ 
$S \cap U \cap \Psi^{-1}(U) \cap \cdots \cap \Psi^{-n_{0}+1}(U)$ and $v \in T_{x} M$ such that $D \Psi^{n_{0}}(x) \cdot v \notin \mathcal{C}\left(\Psi^{n_{0}}(x)\right)$, the angle of $v$ with $T_{x} S$ is bounded away from zero so that its projection has norm larger than $C_{2}^{-1}\|v\|$ for another constant $C_{2}>0$.

The $(1+\alpha)$-contraction of the cone field gives an integer $N \geqslant 1$ satisfying the following.

Lemma 4.22. There exists $N \geqslant 1$ such that, for any $x \in S \cap U \cap \Psi^{-1}(U) \cap \cdots \cap$ $\Psi^{-N+1}(U)$, and any unit vector $u \in T_{x} M$ close to $T_{x} S$, and any unit $w \in \mathcal{C}(x)$,

$$
d\left(D \Psi^{N}(x) \cdot u, T_{\Psi^{N}(x)} S\right) \leqslant \frac{1}{4} \min \left(\left\|D \Psi_{\mid S}^{-N}(x)\right\|^{-\alpha}, 1\right) d\left(u, T_{x} S\right) .
$$

Proof. The proof is similar to the contraction Lemma 2.6: we may choose $v$ such that $u+v$ belongs to $T_{x} S$ and $D \Psi^{N}(x) \cdot v$ is tangent to the fiber of $\pi$ at $\Psi^{N}(x)$. One deduces that

$$
d\left(D \Psi^{N}(x) \cdot u, T_{\Psi^{N}(x)} S\right)=\frac{\left\|D \Psi^{N}(x) \cdot v\right\|}{\left\|D \Psi^{N}(x) \cdot u\right\|} .
$$

The distance $d\left(u, T_{x} S\right)$ is the norm of the projection of $u$ to the fiber of $x$ parallel to $T_{x} S$. It is thus equal to $d\left(v, T_{x} S\right)$ and is larger than

$$
d\left(u, T_{x} S\right) \geqslant C_{2}^{-1}\|v\| .
$$

The $(1+\alpha)$-cone contraction gives, for any unit vector $w \in \mathcal{C}(x)$,

$$
\frac{\left\|D \Psi^{N}(x) \cdot v\right\|}{\|v\|} \leqslant C \lambda^{-N} \min \left(\left\|D \Psi^{N}(x) \cdot w\right\|^{1+\alpha},\left\|D \Psi^{N}(x) . w\right\|\right) .
$$

In particular, if $w$ is the most contracted unit vector in $\mathcal{C}(x)$,

$$
\frac{\left\|D \Psi^{N}(x) \cdot v\right\|}{\left\|D \Psi^{N}(x) \cdot u\right\|} \leqslant C \lambda^{-N} \min \left(\left\|D \Psi^{N}(x) . w\right\|^{\alpha}, 1\right)\|v\| \leqslant C \lambda^{-N} \min \left(\left\|D \Psi_{\mid S}^{-N}(x)\right\|^{-\alpha}, 1\right)\|v\| .
$$

Putting the inequalities together, one gets the announced estimate, provided that $C C_{2} \lambda^{-N} \leqslant \frac{1}{4}$

Working in charts, one can identify the tangent spaces $T_{x} M$ and $T_{x}^{\prime} M$ at points close to each other. Since $D \Psi^{N}$ is $\alpha$-Hölder continuous, there exists a constant $C_{3}>0$ such that, for points $x, x^{\prime}$ close and any unit vector $u$,

$$
\left\|D \Psi^{N}(x) \cdot u-D \Psi^{N}\left(x^{\prime}\right) \cdot u\right\| \leqslant C_{3} d\left(x, x^{\prime}\right)^{\alpha} .
$$

Let us now finish the proof of the addendum. Let us denote $\left(z_{-i}\right)_{0 \leqslant i \leqslant \ell}$ the backward orbit of $z$ by $\Psi^{N}$ in $S \cap U$ : it is infinite $(\ell=\infty)$ or defined for $i$ smaller than some integer $\ell$. We fix $\sigma>0$ (small and independent of $z, z^{\prime}$ ) so that the point $z^{\prime} \in S$ has backward iterates $z_{-i}^{\prime}=\Psi^{-i N}\left(z^{\prime}\right)$ by $\Psi^{N}$ in $S$ whenever the distance $d\left(z_{-i}^{\prime}, z_{-i}\right)$ is smaller than $\sigma$. If $\sigma$ has been chosen small enough, the distance between $z_{-i}$ and $z_{-i}^{\prime}$ is smaller than

$$
d\left(z_{-i}, z_{-i}^{\prime}\right) \leqslant 2^{i} \prod_{j=0}^{i-1}\left\|D \Psi_{\mid S}^{-N}\left(z_{-j}\right)\right\| d\left(z, z^{\prime}\right) .
$$


Let us consider a sequence of unit vectors $u_{-i} \in T S$ at $z_{-i}^{\prime}$ such that $u_{-i}$ is collinear to $D \Psi^{N}\left(z_{-(i+1)}^{\prime}\right) \cdot u_{-(i+1)}$ for each $i$. We denote $v_{-i}$ the unit vector collinear to $D \Psi^{N}\left(z_{-(i+1)}\right) \cdot u_{-(i+1)}$

We estimate inductively the distance between $u_{-i}$ and $T S$. There exists $C_{4}>0$ satisfying

$$
\begin{aligned}
d\left(u_{-i}, T_{z_{-i}} S\right) \leqslant & C_{1}\left\|u_{-i}-v_{-i}\right\|+d\left(v_{-i}, T_{z_{-i}} S\right) \\
\leqslant & 2 C_{1} \frac{C_{3} d\left(z_{-(i+1)}, z_{-(i+1)}^{\prime}\right)^{\alpha}}{\left\|D \Psi^{N}\left(z_{-(i+1)}^{\prime}\right) \cdot u_{-(i+1)}\right\|} \\
& +\frac{1}{4} \min \left(1,\left\|D \Psi_{\mid S}^{-N}\left(z_{-i}\right)\right\|^{-\alpha}\right) d\left(u_{-(i+1)}, T_{z_{-(i+1)}} S\right) \\
\leqslant & C_{4} d\left(z_{-(i+1)}, z_{-(i+1)}^{\prime}\right)^{\alpha}+\frac{1}{4} \min \left(1,\left\|D \Psi_{\mid S}^{-N}\left(z_{-i}\right)\right\|^{-\alpha}\right) d\left(u_{-(i+1)}, T_{z_{-(i+1)}} S\right) .
\end{aligned}
$$

We thus obtain, for any $k \leqslant \ell$,

$$
\begin{aligned}
d\left(u_{0}, T_{z} S\right) \leqslant & \sum_{i=1}^{k} 4^{-i} C_{4} \prod_{j=0}^{i-1}\left\|D \Psi_{\mid S}^{-N}\left(z_{-j}\right)\right\|^{-\alpha} d\left(z_{-i}, z_{-i}^{\prime}\right)^{\alpha} \\
& +4^{-k} \prod_{j=0}^{k-1} \min \left(1,\left\|D \Psi^{-N}\left(z_{-j}\right)\right\|^{-\alpha}\right) d\left(u_{-k}, T_{z_{-k}} S\right) \\
\leqslant & C_{4} d\left(z, z^{\prime}\right)^{\alpha}+\min \left(2^{-k} \frac{d\left(z, z^{\prime}\right)^{\alpha}}{d\left(z_{-k}, z_{-k}^{\prime}\right)^{\alpha}}, 4^{-k}\right)
\end{aligned}
$$

Three cases are possible.

- The backward orbit of $z$ is infinite (i.e., $\ell=\infty$ ), and the distance $d\left(z_{-k}, z_{-k}^{\prime}\right)$ is smaller than $\sigma$ for any $k$. In this case, $k$ can be taken arbitrarily large.

- There is $k \leqslant \ell$ such that $d\left(z_{-k}, z_{-k}^{\prime}\right)$ is of the order of $\sigma$ : for some constant $C_{5}>0$, we have

$$
2^{-k} \frac{d\left(z, z^{\prime}\right)^{\alpha}}{d\left(z_{-k}, z_{-k}^{\prime}\right)^{\alpha}} \leqslant C_{5} d\left(z, z^{\prime}\right)^{\alpha}
$$

- The distance $d\left(z_{-k}, z_{-k}^{\prime}\right)$ is smaller than $\sigma$ for any $k \leqslant \ell$. Moreover, there exist $j \in\{1, \ldots, N\}$ and a point

$$
\tilde{z} \in(S \backslash \Psi(S \cap U)) \cap(S \cap U) \cap \cdots \cap \Psi^{-j}(S \cap U)
$$

such that $\Psi^{j}(\widetilde{z})=z_{-\ell}$. Since $\sigma>0$ has been chosen small, there also exists a point $\widetilde{z}^{\prime}$ in a compact neighborhood of $S \backslash \Psi(S \cap U)$ contained in $V$ such that $\Psi^{j}\left(\widetilde{z}^{\prime}\right)=z_{-\ell}^{\prime}$. Since $S$ is $C^{1+\alpha}$ on $V$, one deduces that there exists $C_{6}>0$ uniform 
such that

$$
d\left(u_{-\ell}, T_{z_{-\ell}} S\right) \leqslant C_{6} d\left(z_{-\ell}, z_{-\ell}^{\prime}\right)^{\alpha} \leqslant C_{6} 2^{\ell} \prod_{j=0}^{\ell-1}\left\|D \Psi_{\mid S}^{-N}\left(z_{-j}\right)\right\|^{\alpha} d\left(z, z^{\prime}\right)^{\alpha}
$$

so that $d\left(u_{0}, T_{z} S\right) \leqslant C_{4} d\left(z, z^{\prime}\right)^{\alpha}+2^{-\ell} C_{6} d\left(z, z^{\prime}\right)^{\alpha}$.

In any case, we have shown that the distance between $T_{z} S$ and $T_{z^{\prime}} S$ is smaller than $C_{7} d\left(z, z^{\prime}\right)^{\alpha}$ for a uniform constant $C_{7}$, which ends the proof of the addendum.

\subsection{Conclusion of the proof of Theorem 4.1}

The graph transform also fixes the space $\operatorname{Lip}_{m, \beta}(K)$, and hence the graph $S$ of the function $h \in \operatorname{Lip}_{m, \beta}$ fixed by $G_{m}$ is a $C^{1}$-submanifold, having the same dimension as $\Sigma$ and containing $K$ in its interior. Note that it can be extended as a submanifold with boundary, still denoted by $S$, by taking the union with $\Sigma \backslash \Sigma_{0}$. By the definition of $G_{m}$, the submanifolds $S$ and $f(S)$ coincide on a neighborhood of $K$. Moreover, $T S_{\mid K}$ is an invariant subbundle transverse to $E^{u u}$, and hence coincides with $E^{c}$ at points of $K$. The proofs of Theorem 4.1 and of the main theorem are now complete.

\section{Consequences}

\subsection{Dynamics in a neighborhood: proof of Corollaries 1.2 and $\mathbf{1 . 3}$}

Under the setting of the main theorem, one considers $0<\delta \ll \varepsilon$ small, and a neighborhood $U$ of $K$. Provided that $U$ is small enough, any point $x$ in the maximal invariant set of $U$ by $f$ has a strong unstable manifold of size $\varepsilon$ which intersects $S$ at some unique point $s(x)$. Moreover, the intersection is transverse, $d(x, s(x))<\delta$, and $s(x)$ belongs to a small neighborhood of $K$ in $S$. In particular, $f(s(x))$ still belongs to the unstable manifold of size $\varepsilon$ of $f(x)$ and to $S$. One deduces that, for any $n \in \mathbb{Z}$, one has

$$
f^{n}(s(x))=s\left(f^{n}(x)\right) .
$$

Taking $n$ arbitrarily large, the distance $d(x, s(x))$ is exponentially smaller than $d\left(f^{n}(x), s\left(f^{n}(x)\right)\right)$, which is bounded by $\delta$. This proves that $x=s(x)$. The maximal invariant set of $U$ is thus contained in $S$. This proves Corollary 1.2.

Corollary 1.3 is obtained by applying the main theorem to $f$ and $f^{-1}$, respectively. The submanifold $S$ is built as the intersection of two locally invariant submanifolds $S^{c s}, S^{c u}$ containing $K$ and tangent to $E^{s s} \oplus E^{c}$ and $E^{c} \oplus E^{u u}$, respectively.

\subsection{Robustness of the submanifold: proof of Corollary 1.4}

The definition of the graph transform and the results of $\S \S \S 4.2 .2,4.3 .1$ and 4.3 .2 allow some flexibility: once the space $\operatorname{Lip}_{m, \beta}$ and the function $\varphi_{m}$ have been chosen, the set $K$ is not considered any more, and the graph transform may be modified into a map which is $C^{1}$-close to the initial transformation. In particular, one can replace $f$ by any diffeomorphism $g$ that is $C^{1}$-close to $f$. The map $\Psi_{m}$ introduced in (12) can then be modified as a $C^{\infty}$-map

$$
\Psi_{m, g}: z \mapsto \Theta\left(\varphi_{m} \circ g^{-1}(z), g^{-1}(z)\right)
$$


which defines a new graph transform, producing a new $C^{1}$-submanifold $S_{g}$. By considering a small neighborhood $U$ of $K$ and arguing as for the proof of Corollary 1.2, one gets that the maximal invariant set of $g$ in $U$ is contained in $S_{g}$.

The graphs $S_{g}$ and $g\left(S_{g}\right)$ coincide over an open set of $\Sigma_{0}$ which is independent from the diffeomorphism $g$ (the points where $\varphi_{m}$ equals 1 in $\S 4.2 .2$ ). This proves that the restriction of the graph $S_{g}$ to this open set defines a submanifold $S_{g}^{\prime}$ which depends continuously on $g$ for the $C^{1}$-topology, is contained in $S_{g} \cap g\left(S_{g}\right)$, and such that $S_{f}^{\prime}$ contains a neighborhood of $K$ in $S_{f}$.

Provided that two diffeomorphisms $g_{1}$ and $g_{2}$ are close enough for the $C^{1}$-topology, the fixed points in the space $\operatorname{Lip}_{m, \beta}$ are close enough. This proves that the graphs $S_{g_{1}}$ and $S_{g_{2}}$ are $C^{0}$ close. Both are tangent to some thin cone fields obtained by iteration (see $\S 4.3 .2$ ). When $g_{1}, g_{2}$ are $C^{1}$-close, these cone fields are close, and hence $S_{g_{1}}, S_{g_{2}}$ are $C^{1}$-close. This proves that $g \mapsto S_{g}$ varies continuously for the $C^{1}$-topology and this ends the proof of Corollary 1.4 .

\subsection{Higher regularity}

5.3.1. $C^{r}$-regularity of the center manifold: proof of Corollary 1.5. Let us continue the proof of $\S 4$ under the assumption that $f$ is $C^{r}$ and that the partially hyperbolic set $K$ is $r$-normally hyperbolic for some $r>1$. The argument to prove that $S$ is $C^{r}$ follows the ideas of the $C^{r}$-section theorem in [12] (although we were not able to apply this theorem directly since the map $\Phi_{m}$ which is used for the graph transform is not defined on an invariant domain).

Note that, by Remarks 4.5 and 4.12 , one can assume that the submersion $\pi: T \rightarrow$ $\Sigma_{0}$ is smooth, and that the graph transform $\Psi:=\Psi_{m}$ introduced in $\S 4.2 .2$ is $C^{r}$. Proposition 4.14 and Addendum 4.16 provide us with a cone field $\mathcal{C}:=\mathcal{C}_{\beta}^{h}$ on $T$ of dimension $d$ which is transverse to the submersion $\pi$ and $r$-contracted by $\Psi$. Moreover, $S$ is tangent to $\mathcal{C}$. Let us consider the domain $U:=U_{m}$ and the open set $V:=V_{m}$; then Proposition 4.19 applies. If $r=1+\alpha$ with $\alpha \in(0,1)$, Addendum 4.21 proves that the submanifold $S$ is $C^{r}$ also. It remains thus to consider the case $r \geqslant 2$.

We introduce the Grassmannian bundle $p: \widehat{T} \rightarrow T$ of $d$-dimensional tangent subspaces of $T$. Since $r \geqslant 2$, Addendum 4.21 proves that $S$ is the graph of a $C^{1,1}$ map. Consequently, the tangent spaces to $S$ define a Lipschitz graph $\widehat{S}$ of the fibration $\widehat{\pi}:=\pi \circ p: \widehat{T} \rightarrow$ $\Sigma_{0}$. The preimages $\widehat{U}:=p^{-1}(U)$ and $\widehat{V}:=p^{-1}(V) \cap \widehat{S}$ are open subsets of $\widehat{T}$ and $\widehat{S}$, respectively. The tangent map $D \Psi$ induces a $C^{r-1}$ map $\widehat{\Psi}: \widehat{U} \rightarrow \widehat{T}$, and the two first properties of the Proposition 4.19 hold.

Since $\widehat{S}$ is Lipschitz, the angle between the tangent space to $\widehat{S}$ and the tangent space to the fibers of $p$ is uniformly bounded away from zero. The unit tangent vectors to $\widehat{S}$ are thus contained in a compact set of vectors $v$ satisfying $D p(v) \neq 0$. One can apply Proposition 2.12 and get a continuous cone field $\widehat{\mathcal{C}}$ on $\widehat{T}$, of dimension $d$, which is transverse to the fibration $\widehat{\pi}$ and $(r-1)$-contracted by $\widehat{\Psi}$. Moreover, one can require that $\widehat{S}$ is tangent to $\widehat{\mathcal{C}}$; indeed the collection of unit vectors in the tangent sets of $\widehat{S}$ is contained in a compact set of vectors $v$ satisfying $D p(v) \in \mathcal{C} \backslash\{0\}$.

Proposition 4.19 and Addendum 4.21 now imply that $\widehat{S}$ is a $C^{1, \alpha}$-submanifold of $\widehat{T}$, and hence that $S$ is $C^{2, \alpha}$, where $\alpha=\min (1, r-2)$. For any integer $k \leqslant r-1$, one can repeat 
this argument inductively $k$ times and conclude that $S$ is $C^{k, \alpha}$, where $\alpha=\min (1, r-k-$ 1). This proves that $S$ is $C^{r}$, and gives the first item of Corollary 1.5.

If $f$ is $C^{r}$ for some $r>1$, and if we only assume that $K$ is partially hyperbolic, the continuity of the tangent map and the compactness of the unit bundle inside the central bundle $E^{c}$ on $K$ imply that $K$ is $(1+\alpha)$-normally hyperbolic for some $\alpha>0$ small. One deduces that $S$ can be chosen $C^{1, \alpha}$, proving the second item of Corollary 1.5.

5.3.2. Smoothing the submanifold: proof of Proposition 1.6. Let $S^{\prime} \subset \operatorname{int}(S \cap$ $f(S)$ ) be a submanifold with boundary which contains $K$ in its interior. Let $U$ be a small neighborhood of $K$. Let us consider a $C^{\infty}$-diffeomorphism $g_{0}$ and a $C^{\infty}$-submanifold $S_{g}$ close to $f$ and $S$ for the $C^{1}$-topology. The image $g_{0}\left(S_{g}\right)$ is $C^{1}$-close to $S$, and in particular is arbitrarily $C^{1}$-close to $S_{g}$ in a neighborhood of $S^{\prime}$. More precisely, there exist $S_{g}^{\prime} \subset S_{g}$ and $\widetilde{S}_{g}^{\prime} \subset g_{0}\left(S_{g}\right)$ which both project on $S^{\prime}$ by $\pi$. One can thus consider a diffeomorphism $\tau$ of a neighborhood of $S^{\prime}$, which is a translation along each curve $\pi^{-1}(x)$ of the tubular neighborhood $T$ and which maps $\widetilde{S}_{g}^{\prime}$ on $S_{g}^{\prime}$. Since $\pi$ is $C^{\infty}$, and since $S_{g}^{\prime}$ and $\widetilde{S}_{g}$ are $C^{\infty}$-submanifolds which are $C^{1}$-close, one deduces that $\tau$ is a $C^{\infty}$-diffeomorphism which is $C^{1}$-close to the identity. It can be extended as a smooth diffeomorphism of $M$. The $C^{\infty}$ diffeomorphism $g:=\tau \circ g_{0}$ is $C^{1}$-close to $f$, and by construction $S_{g}^{\prime} \subset S_{g} \cap g\left(S_{g}\right)$. Arguing as in the proof of Corollary 1.2, one shows that the maximal invariant set $\Lambda_{g}$ of $U$ is contained in $S_{g}$. This gives the proposition.

\subsection{Consequences when the center dimension equals 1 or 2}

5.4.1. One-dimensional center bundle: proof of corollary 1.7. The arguments for one-dimensional invertible systems are classical, and we only recall the main ideas. Let $K$ be a compact invariant set endowed with a partially hyperbolic structure whose center bundle is one dimensional, and assume that $K$ has no strong connection. By Corollary 1.3, the set $K$ is contained in a family of curves and circles $\gamma_{1}, \ldots, \gamma_{k}$ that are tangent at $E^{c}$ at points of $K$ and such that, for any point $x \in \Gamma:=\cup_{i} \gamma_{i}$ close to $K$, the image of $x$ is still contained in $\Gamma$. Any minimal subset $C$ of $K$ is either a periodic circle, a periodic orbit, or a Cantor set. In the third case, the orbit of any point $x \in \Gamma$ close to $C$ accumulates on $\Lambda$ in the past or in the future. In particular, there exist at most finitely many non-periodic minimal sets, and any orbit in $K$ accumulates in the future and in the past to minimal sets.

One can $C^{1}$-approximate $f$ by a diffeomorphism $g$ whose periodic orbits are hyperbolic and whose minimal sets are limit for the Hausdorff topology of periodic orbits. By Corollary 1.4, the maximal invariant set $\Lambda_{g}$ for $g$ in a neighborhood $U$ of $K$ is still contained in a one-dimensional $C^{1}$-submanifold $\Gamma_{g}$, and the dynamics of $g$ on $\Lambda_{g}$ satisfies the same properties as $(K, f)$. However, for any minimal set $C \subset \Lambda_{g}$, there exists a periodic orbit $O$ contained in an arbitrarily small neighborhood of $C$. One deduces that $O$ is contained in $\Gamma_{g}$. Since the non-periodic minimal sets are isolated in $\Gamma_{g}$ from the periodic orbits, they cannot exist for $g$. There are at most finitely many periodic orbits since they are hyperbolic. This gives the conclusion of Corollary 1.7.

5.4.2. Two-dimensional center bundle: proof of Corollary 1.8. By Corollary 1.4, for any diffeomorphism $g$ that is $C^{1}$-close to $f$, the maximal invariant 
set $\Lambda_{g}$ in $U$ has no strong connection, and is contained in a locally invariant $C^{1}$-surface $\Sigma_{g}$. Let us make two remarks.

- $\Sigma_{g}$ is in general not a boundaryless compact manifold, but some known results for surface dynamics which only involve local arguments extend to this setting.

- If $h$ is a $C^{1}$-perturbation of the restriction $g_{\mid \Sigma_{g}}$ supported on an arbitrarily small neighborhood of $\Lambda_{g}$, then it extends as a diffeomorphism of $M$ that is close to $f$ for the $C^{1}$-topology such that $\Sigma_{g}$ contains $\Lambda_{h}$ and is locally invariant in a neighborhood of this set. Indeed, one can decompose $h=\varphi \circ g_{\mid \Sigma_{g}}$ where $\varphi$ is a diffeomorphism of $\Sigma_{g}$ which is $C^{1}$-close to the identity and supported inside a small neighborhood of $\Lambda_{f}$. Since $\varphi$ is isotopic to the identity among diffeomorphisms of $\Sigma_{g}$ close to the identity with compact support, one can extend $\varphi$ to a diffeomorphism of $M$ close to the identity.

Since $U$ is a filtrating set, the intersection $\mathcal{R}(g) \cap U$ is a union of chain-recurrence classes $C$.

Lemma 5.1. Let us assume that the first case of the Corollary 1.8 does not hold. Taking $g$ in a dense $G_{\delta}$-subset of $\mathcal{U}$, the center bundle over any non-trivial chain-recurrence class $C \subset U$ has a dominated splitting $E_{\mid C}^{c}=E_{1}^{c} \oplus E_{2}^{c}$, i.e., there exists $N \geqslant 1$ such that, for any $x \in C$ and any $u, \in E_{1}^{c}(x), v \in E_{2}^{c}(x)$, one has $\left\|D g^{N}(u)\right\| \leqslant \frac{1}{2}\left\|D g^{n}(v)\right\|$.

Proof. Taking $g$ in a dense $G_{\delta}$-subset of $\mathcal{U}$, one can assume that any chain-recurrence class $C$ which is not a periodic orbit is the limit of a sequence of hyperbolic periodic orbits $\left(O_{n}\right)$ (see [4]), and then argue as in [19].

A result by Pliss (see [19, Theorem 2.1]) asserts that by perturbation of $g_{\mid \Sigma_{g}}$ one can turn one of the periodic orbits $O_{n}$ to be a saddle inside $\Sigma_{g}$. By a standard Baire argument this implies that $C$ is also the limit of hyperbolic periodic orbits whose stable and unstable spaces intersect $E^{c}$ along one-dimensional subspaces, inducing an invariant splitting $E_{1}^{c} \oplus E_{2}^{c}$ of $E^{c}$ over the union of the orbits $O_{n}$. If this splitting is not dominated, one can create by perturbation of $g_{\mid \Sigma_{g}}$ a homoclinic tangency for one of these saddles (see [11]). This perturbation may be extended as a diffeomorphism of $M$, and the first case of the corollary holds. Otherwise, there exists a dominated splitting on the union of the $O_{n}$, and hence on their closure and on $C$ (see [2, Appendix B.1.1]).

As a consequence, the set $\mathcal{R}(g) \cap U$ decomposes into finitely many isolated periodic orbits and a set whose center bundle has a dominated splitting. The previous argument shows that (up to reducing $U$ and replacing $f$ by a diffeomorphism $C^{1}$-close) one can restrict to the case the center bundle of $\Lambda_{f}$ has a dominated splitting $E^{c}=E_{1}^{c} \oplus E_{2}^{c}$. This also holds for any diffeomorphism $g$ in a neighborhood $\mathcal{U}$.

By Proposition 1.6, one can consider $g \in \mathcal{U}$ such that $\Sigma_{g}$ and $g_{\mid \Sigma_{g}}$ are smooth. By perturbation of $g_{\mid \Sigma_{g}}$, one can furthermore assume that all the periodic orbits in $\Sigma_{g}$ are hyperbolic, and that there do not exist minimal sets in $\Sigma_{g}$ which are a finite union of circles that are normally hyperbolic. One can now apply the result of [19] (once again, the argument involves only the dynamics in a neighborhood of $K$, and the diffeomorphism $g$ may be only defined on a neighborhood of $K$ ). 
Theorem (Pujals-Sambarino). Consider a $C^{2}$-diffeomorphism $g$ of a surface $S$, and an invariant compact set $K$ with a dominated splitting $T S_{\mid K}=E_{1}^{c} \oplus E_{2}^{c}$ such that each periodic orbit in $K$ is a hyperbolic saddle and $K$ does not contain a minimal set which is a finite union of circles that is normally hyperbolic. Then $K$ is a hyperbolic set.

For the dynamics of $g_{\mid \Sigma_{g}}$, the set $\mathcal{R}(g) \cap \Lambda_{g}$ is thus the union of sinks, of sources, and of a saddle compact set $K$. In particular, the number of sinks and sources is finite. By Smale's spectral decomposition theorem, the set $K$ decomposes into finitely many transitive subsets, as announced by Corollary 1.8 .

\subsection{Invariant foliations for surface hyperbolic sets: proof of Corollary 1.11}

Let $f$ be a $C^{2}$-surface diffeomorphism and $K$ be an invariant compact set which is hyperbolic. In particular, $E^{u}$ is 2-dominated by $E^{s}$ for $f^{-1}$, and by Lemma 2.10 there exists a continuous cone field $\mathcal{C}$ of dimension 1 which is 2-contracted in a neighborhood of $K$ : we have $E^{u}(x) \subset \mathcal{C}(x)$ at each $x \in K$.

Let $\widehat{M}$ denote the projectivization of the tangent bundle $T M$ (that is, the Grassmannian bundle of one-dimensional tangent spaces) and $p: \widehat{M} \rightarrow M$ the natural projection. The tangent dynamics $D f$ induces a $C^{1}$-diffeomorphism $\widehat{f}$ of $\widehat{M}$. Since the unstable bundle $E^{u}$ on $K$ is one dimensional, it induces a point $\widehat{x}$ in each fiber $p^{-1}(x)$ with $x \in K$, defining a lift $\widehat{K} \subset \widehat{M}$ of $K$ which is invariant by $\widehat{f}$. Since $x \mapsto E^{u}(x)$ is continuous, the set $\widehat{K}$ is compact. By Proposition 2.15 and Remark 2.14, there exist neighborhoods $\widehat{U}$ of $\widehat{K}$ and $U=p(\widehat{U})$ of $K$ and a contracted continuous cone field $\widehat{\mathcal{C}}$ of dimension 2 on $\widehat{U}$ that is transverse to $p$. By Lemma 2.10, this proves that $\widehat{K}$ has a dominated splitting $T \widehat{M}_{\mid} \widehat{K}=\widehat{E} \oplus \widehat{F}$ where $\widehat{F}$ has two-dimensional spaces. Since the tangent spaces to the fibers of $p$ at points of $\widehat{K}$ are preserved by $\widehat{f}$ and since the fibers of $p$ are contracted by $\widehat{f}$ (see Proposition 2.11), one deduces that $\widehat{K}$ is partially hyperbolic with a dominated splitting $T \widehat{M}_{\mid \widehat{K}}=\widehat{E}^{s s} \oplus \widehat{E}^{c}$. The projection $D p$ is an isomorphism between $\widehat{E}^{c}$ and $T M_{\mid K}$.

Since the fibers of $p$ are invariant by $\widehat{f}$ and tangent to $\widehat{E}^{s s}$ at points $\widehat{x}$ of $\widehat{K}$, one deduces that each strong stable manifold $W^{s s}(\widehat{x})$ is contained in $p^{-1}(p(x))$. In particular, it intersects $\widehat{K}$ in a single point and the main theorem applies.

Let $S \subset \widehat{M}$ be a locally invariant $C^{1}$ surface containing $\widehat{K}$ and tangent to $\widehat{E}^{c}$ at points of $\widehat{K}$. The projection $p: S \rightarrow M$ is a local diffeomorphism, injective on $\widehat{K}$, and hence injective on a neighborhood of $\widehat{K}$ : reducing $S$ if necessary, $p$ is a diffeomorphism between $S$ and a neighborhood $U$ of $K$. Moreover, $U$ is endowed with a $C^{1}$ line field $\mathcal{L}: x \mapsto$ $p^{-1}(x) \cap S$ which is locally invariant by $D f$ by construction. This line field uniquely integrates as a foliation $\mathcal{F}^{u}$ on $U$ that is locally invariant on a neighborhood of $K$ and that is tangent to $E^{u}$ at points of $K$. In particular, the leaves $\mathcal{F}_{x}^{u}$ at points $x \in K$ contain the local stable manifolds of $x$.

If $f$ is $C^{r}$, with $r>2$, then $\widehat{f}$ is $C^{r-1}$, and $S$ can be chosen $C^{1, \alpha}$ for some $\alpha>0$ by Corollary 1.5. In particular, the line field $\mathcal{L}$ and the foliation $\mathcal{F}^{s}$ are $C^{1, \alpha}$.

Acknowledgements. The second author is grateful to Enrique Pujals and Geneviève Raugel for their comments related to this work. We thank also the anonymous referees for their remarks, which improved the presentation of the text. This work was partially 
supported by the ANR project DynNonHyp BLAN08-2 313375 and by the Balzan Research Project of J. Palis. The authors acknowledge the IFUM and the CMAT (Montevideo), where part of this text was written.

\section{References}

1. R. Abraham and J. Robbin, Transversal mappings and flows (W. A. Benjamin, Inc., New York-Amsterdam, 1967).

2. C. Bonatti, L. Díaz And M. Viana, Dynamics Beyond Uniform Hyperbolicity (Springer, Berlin, 2004).

3. S.-N. Chow, W. Liu, Y. Yi And Yingfei, Center manifolds for invariant sets, J. Differential Equations 168 (2000), 355-385.

4. S. Crovisier, Periodic orbits and chain-transitive sets of $C^{1}$-diffeomorphisms, Publ. Math. Inst. Hautes Études Sci. 104 (2006), 87-141.

5. S. Crovisier, Partial hyperbolicity far from homoclinic bifurcations, Adv. Math. 226 (2011), 673-726.

6. S. Crovisier and N. Gourmelon, Stabilisation of homoclinic tangencies in higher dimension. In preparation.

7. S. Crovisier and E. R. Pujals, Essential hyperbolicity versus homoclinic bifurcations. Invent. Math. to appear.

8. S. Crovisier, E. R. Pujals and M. Sambarino, Hyperbolicity of extremal bundles. In preparation.

9. W. DE MELo, Structural stability of diffeomorphisms on two-manifolds, Invent. Math. 21 (1973), 233-246.

10. N. Gourmelon, Adapted metrics for dominated splittings, Ergodic Theory Dynam. Systems 27 (2007), 1839-1849.

11. N. Gourmelon, Generation of homoclinic tangencies by $C^{1}$-perturbations, Discrete Contin. Dyn. Syst. 26 (2010), 1-42.

12. M. Hirsch, C. Pugh And M. Shub, Invariant Manifolds, Lecture Notes in Mathematics, volume 583 (Springer-Verlag, Berlin, 1977).

13. R. MAÑÉ, Hyperbolicity, sinks and measure in one dimensional dynamics, Commun. Math. Phys. 100 (1985), 495-524. and Commun. Math. Phys. 112 (1987), 721-724.

14. S. Newhouse, Diffeomorphisms with infinitely many sinks, Topology 13 (1974), 9-18.

15. S. Newhouse, The abundance of wild hyperbolic sets and nonsmooth stable sets for diffeomorphisms, Publ. Math. Inst. Hautes Études Sci. 50 (1979), 101-151.

16. J. Palis And F. Takens, Hyperbolicity and Sensitive Chaotic Dynamics at Homoclinic Bifurcations, Cambridge Studies in Advanced Mathematics, vol. 35 (Cambridge University Press, 1993).

17. J. Palis and M. Viana, High dimension diffeomorphisms displaying infinitely many periodic attractors, Ann. of Math. 140(1) (1994), 207-250.

18. E. R. Pujals, On the density of hyperbolicity and homoclinic bifurcations for 3Ddiffeomorphisms in attracting regions, Discrete Contin. Dyn. Syst. 16 (2006), 179-226.

19. E. R. Pujals And M. Sambarino, Homoclinic tangencies and hyperbolicity for surface diffeomorphisms, Ann. of Math. 151 (2000), 961-1023.

20. E. R. Pujals and M. Sambarino, Density of hyperbolicity and tangencies in sectional dissipative regions, Ann. Inst. H. Poincaré 26 (2009), 1971-2000.

21. N. Romero, Persistence of homoclinic tangencies in higher dimensions, Ergodic Theory Dynam. Systems 15 (1995), 735-757. 
22. L. Wen, Generic Diffeomorphisms away from homoclinic tangencies and heterodimensional cycles, Bull. Braz. Math. Soc. 35 (2004), 419-452.

23. S. Wiggins, Normally Hyperbolic Invariant Manifolds in Dynamical Systems, Applied Mathematical Sciences, vol. 105 (Springer-Verlag, 1994). 